\title{
Enhancing sensitivities to long-lived particles with high granularity calorimeters at the LHC
}

\author{
Jia Liu, ${ }^{a}$ Zhen Liu, ${ }^{b}$ Lian-Tao Wang ${ }^{a, c}$ and Xiao-Ping Wang ${ }^{d}$ \\ ${ }^{a}$ Enrico Fermi Institute, University of Chicago, \\ Chicago, IL 60637, U.S.A. \\ ${ }^{b}$ Maryland Center for Fundamental Physics, Department of Physics, University of Maryland, \\ College Park, MD 20742, U.S.A. \\ ${ }^{c}$ Department of Physics, University of Chicago, \\ Chicago, IL 60637, U.S.A. \\ ${ }^{d}$ High Energy Physics Division, Argonne National Laboratory, \\ Argonne, IL 60439, U.S.A. \\ E-mail: liuj1@uchicago.edu, zliuphys@umd.edu, liantaow@uchicago.edu, \\ xia.wang@anl.gov
}

ABSTRACT: The search for long-lived particles (LLP) is an exciting physics opportunity in the upcoming runs of the Large Hadron Collider. In this paper, we focus on a new search strategy of using the High Granularity Calorimeter (HGCAL), part of the upgrade of the CMS detector, in such searches. In particular, we demonstrate that the high granularity of the calorimeter allows us to see "shower tracks" in the calorimeter, and can play a crucial role in identifying the signal and suppressing the background. We study the potential reach of the HGCAL using a signal model in which the Standard Model Higgs boson decays into a pair of LLPs, $h \rightarrow X X$. After carefully estimating the Standard Model QCD and the misreconstructed fake-track backgrounds, we give the projected reach for both an existing vector boson fusion trigger and a novel displaced-track-based trigger. Our results show that the best reach for the Higgs decay branching ratio, $\mathrm{BR}(h \rightarrow X X)$, in the vector boson fusion channel is about $\mathcal{O}\left(10^{-4}\right)$ with lifetime $c \tau_{X} \sim 0.1-1$ meters, while for the gluon gluon fusion channel it is about $\mathcal{O}\left(10^{-5}-10^{-6}\right)$ for similar lifetimes. For longer lifetime $c \tau_{X} \sim 10^{3}$ meters, our search could probe $\operatorname{BR}(h \rightarrow X X)$ down to a few $\times 10^{-4}\left(10^{-2}\right)$ in the gluon gluon fusion (vector boson fusion) channels, respectively. In comparison with these previous searches, our new search shows enhanced sensitivity in complementary regions of the LLP parameter space. We also comment on many improvements can be implemented to further improve our proposed search.

Keywords: Beyond Standard Model, Higgs Physics

ArXiv EPrint: 2005.10836 


\section{Contents}

1 Introduction 1

2 Analysis framework $\quad 3$

2.1 Signal model: long-lived particles from Higgs decay 3

2.2 Modeling the HGCAL detector 4

2.3 Signal and background generation 5

2.3.1 The long-lived particle signal 5

2.3.2 SM QCD background 5

2.3.3 Fake-track background 6

$\begin{array}{lll}2.4 & \text { Triggering strategy } & 7\end{array}$

3 The kinematics of signal and backgrounds $\quad 7$

$\begin{array}{ll}3.1 & \text { The displaced vertex fitting variables }\end{array}$

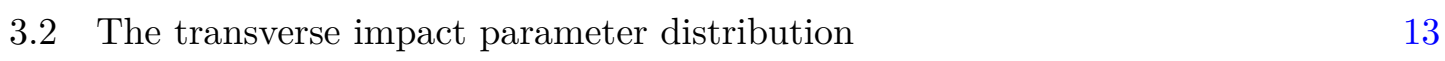

$\begin{array}{lll}3.3 & \text { Correlations between the selection cuts } & 14\end{array}$

$\begin{array}{lll}4 & \text { The results } & 17\end{array}$

$\begin{array}{lll}4.1 & \text { Cut efficiencies } & 17\end{array}$

$\begin{array}{ll}4.2 \text { The reach } & 19\end{array}$

5 Conclusion $\quad 20$

$\begin{array}{ll}\text { A Supportive figures and tables } & 22\end{array}$

\section{Introduction}

Models of new physics beyond the Standard Model (BSM) often predict the existence of long-lived particles (LLPs), giving rise to distinct signatures at colliders (see [1] for a recent review). There have been many searches for LLPs at the ATLAS, CMS, and LHCb experiments at the Large Hadron Collider (LHC). The signatures of the LLP depend on its charge, lifetime, and decay products. Accordingly, various search strategies and detection techniques can be used, including the non-prompt photon detection using the electromagnetic (EM) calorimeter [2,3], the disappearing track searches based on the tracking system [4-6], and the displaced leptons or lepton jets searches based on the tracking system [7-13], the calorimeter [14, 15], as well as the muon system [16-18]. Many new search targets and strategies for the LLPs based on the LHC experiment have also been proposed [19-27, 27-78].

In this work, we focus on a new sub-detector HGCAL, a highly granular and siliconbased calorimeter, which is the Phase-2 upgrade of the CMS endcap calorimeter [79]. It 

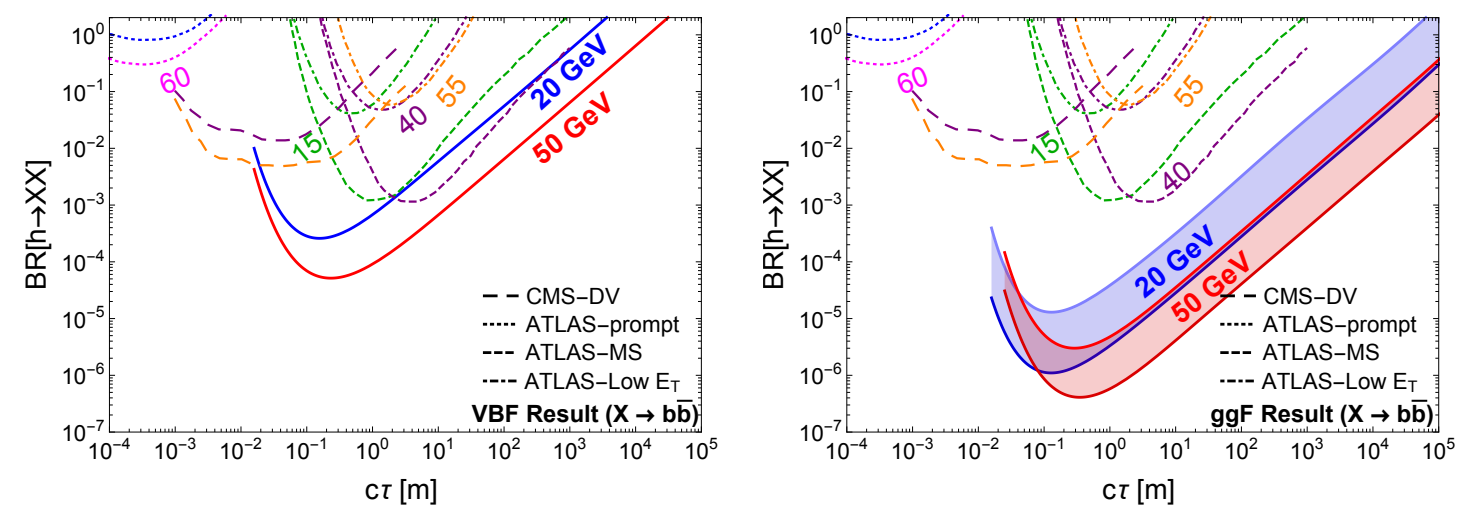

Figure 1. The projected sensitivity for Higgs decays to long-lived particles with VBF trigger (left panel) and a displaced track trigger for the ggF channel (right panel) at the HL-LHC $\left(3 \mathrm{ab}^{-1}\right)$ as a function of proper lifetime of $X$ using our proposed HGCAL LLP search. We consider two scenarios of the displaced track trigger. The solid line on the top of the shaded region corresponds to the reach with a trigger requirement of $H_{T}>100 \mathrm{GeV}$, while the solid line on the bottom of the shaded region is obtained without such additional requirement. The existing limits for $\mathrm{BR}(h \rightarrow X X)$ from ATLAS Run 2 searches based on prompt VH [80] (dotted), the muon spectrometer [18] (dashed), the calorimeter [14] (dot-dashed), with integrated luminosity of $36 \mathrm{fb}^{-1}$, and the CMS search based on displaced vertex in the tracker system [13] (long dashed) with integrated luminosity of $132 \mathrm{fb}^{-1}$, are also shown for comparison. The numbers on different colored lines indicate the mass of the LLP in units of $\mathrm{GeV}$ for the corresponding searches.

consists of a sampling calorimeter with silicon and scintillators as active material, including both the electromagnetic and the hadronic sections with unprecedented fine segmentation. In particular, each section consists of silicon cells of size $\left(0.5-1 \mathrm{~cm}^{2}\right)$ and the remainder of the hadron calorimeter will use highly-segmented plastic scintillators of size $(4-30$ $\mathrm{cm}^{2}$ ) [79]. It has an intrinsic high-precision timing capability from silicon sensors with a resolution of $\sim 25 \mathrm{ps}$. Due to its fine transverse granularity, the HGCAL has an angular resolution of about $5 \times 10^{-3}$ radians for electromagnetic shower with $p_{T}>20 \mathrm{GeV}$, after taking into account the broadening effect from the shower. The HGCAL can handle different LLPs signatures. It also serves as a semi-forward detector different from most LLP studies at LHC main detectors that are mainly based on central detectors. ${ }^{1}$

We carefully simulated and estimated the SM background for generic LLP signals, which contains prompt and displaced QCD background and non-prompt misconnected fake-track background. Based on these, we design a set of cuts that take advantage of the unique features of the signal and the capabilities of the HGCAL detector. We use a signal model in which scalar LLPs $(X)$ are produced from SM Higgs decay $(h \rightarrow X X)$. This simple model is quite representative [81], covering a broad range of new physics scenarios, such as the hidden valley models [82-84], and more recent proposals motivated by neutral naturalness [85-90]. Two production channels of SM Higgs are considered. One is the vector boson fusion (VBF) channel, together with the existing VBF trigger. The other

\footnotetext{
${ }^{1}$ For the consideration of non-pointing photon at HGCAL for the triggering, see [77].
} 

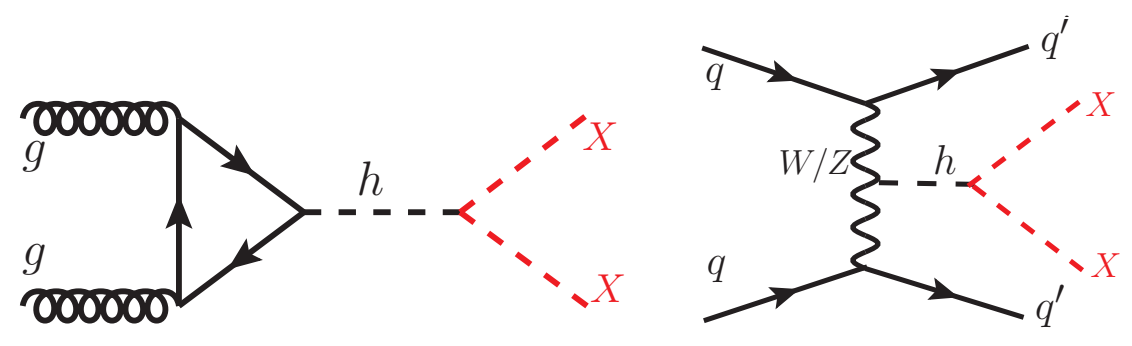

Figure 2. The processes of producing the long-lived particle $X$ from SM Higgs decay considered in this study. Left panel: gluon-gluon fusion Higgs production. Right panel: vector boson fusion Higgs production.

is the gluon-gluon fusion ( $\mathrm{ggF}$ ) channel with a potential displaced track trigger, enabled by new trigger considerations from the tracker and HGCAL. The sensitivity of HL-LHC is given as a function of the proper lifetime of $X$, shown in figure 1 . The best reach for VBF channel is about $\mathrm{BR}(h \rightarrow X X) \sim \mathcal{O}\left(10^{-4}\right)$ with a lifetime of $c \tau_{X} \sim 0.1-1$ meters, while for the ggF channel it is about $\mathrm{BR}(h \rightarrow X X) \sim \mathcal{O}\left(10^{-5}-10^{-6}\right)$ for similar lifetime. Alternatively, for an LLP with $c \tau_{X} \sim 10^{3}$ meters, the HGCAL based search should be able to probe $\operatorname{BR}(h \rightarrow X X)$ down to a few $\times 10^{-4}\left(10^{-2}\right)$ in the $\mathrm{ggF}$ (VBF) channels, respectively.

The paper is organized as follows. In section 2.1, we discuss the signal model and the trigger considerations for the signal. In section 2.3, we describe signal and background generation. In section 3, the distributions of kinematic variables are discussed, and the corresponding cuts are applied. Finally, we show our results in section 4 and conclude in section 5 .

\section{Analysis framework}

\subsection{Signal model: long-lived particles from Higgs decay}

To demonstrate the potential of our proposed search, we use a signal model in which the LLP couples to the SM through the Higgs portal. For $m_{X}<m_{h} / 2$, the LLP will be produced through the Higgs boson decay

$$
h \rightarrow X X
$$

We assume $X$ is a neutral and meta-stable scalar which will further decay via $X \rightarrow \bar{b} b$. The free parameters in this simplified model are mass $m_{X}$, lifetime $c \tau_{X}$, and the decay branching ratio $\mathrm{BR}(h \rightarrow X X)$.

We consider two Higgs production channels, namely, the VBF production and $\operatorname{ggF}$ production, shown in figure 2. The VBF channel is motivated by the possibility of using an existing VBF trigger that does not rely on the properties of the LLP. In the ggF channel, we will explore the physics potential of using displaced track triggers after LHC Phase-2 upgrades, e.g., ref. [91]. Since the metastable particle $X$ is neutral, it does not leave a track as it travels through the detector. Subsequently, $X$ decays to $\bar{b} b$. For our work, 


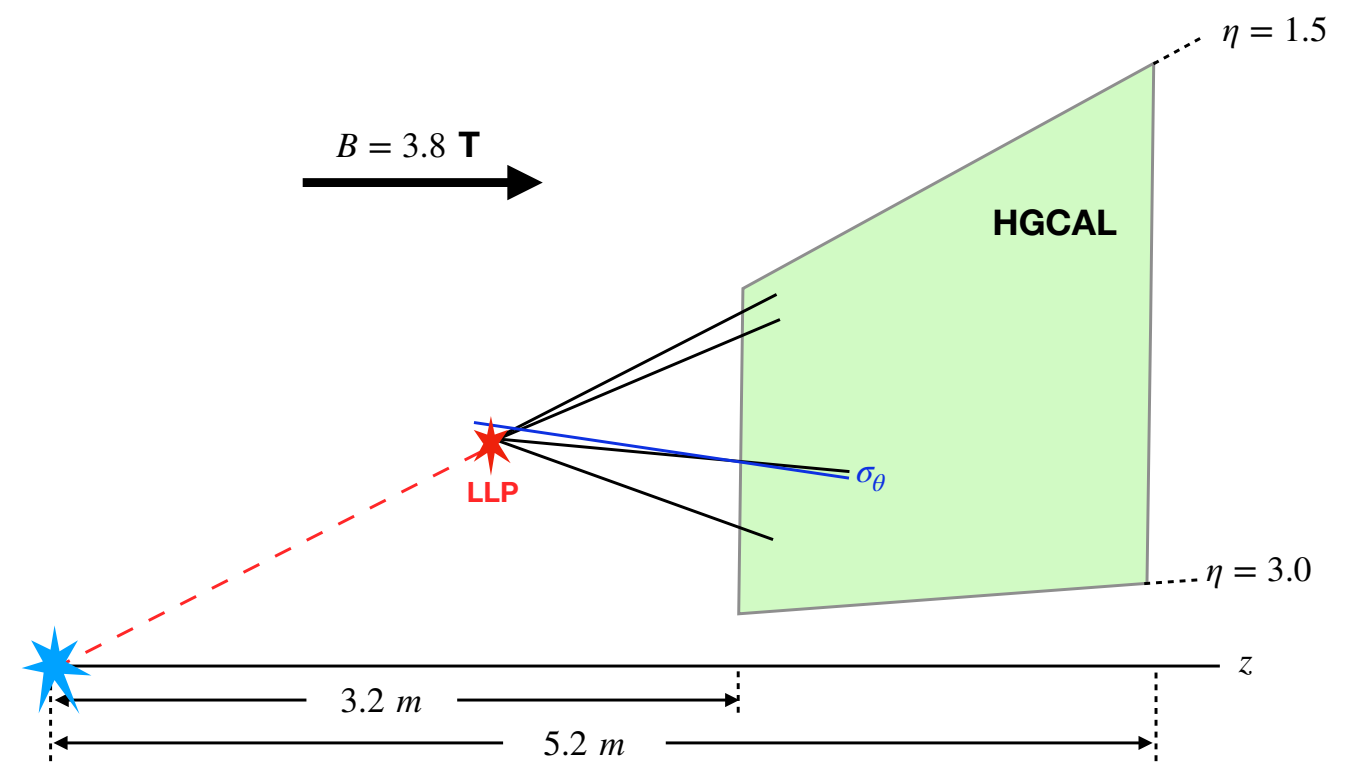

Figure 3. A schematic drawing for the decay products of the long-lived particle arriving the HGCAL. The direction of the momentum of the decay products can be measured by the HGCAL with an angular resolution of $\sigma_{\theta}$, resulting in an error in reconstructing the displaced vertex.

we do not use the tagging information of whether the jets are initiated by heavy or light flavor quarks. ${ }^{2}$

\subsection{Modeling the HGCAL detector}

Our study focuses on the potential of the LLP search of the HGCAL detector [79]. Due to the novelty of the detector and the signature, we cannot perform a full-fledged detector simulation. Instead, we make assumptions based upon the HGCAL performance document. We describe here the relevant detector parameters used in our study.

The HGCAL detector locates at $|z|=3.2 \mathrm{~m}$ and extends to $|z|=5.2 \mathrm{~m}$. The angular coverage of the detector is $1.5<|\eta|<3.0$. Its stand-alone angular resolution on the shower direction is taken to be $\sigma_{\theta} \sim 5 \times 10^{-3}$ radians, with possible improvement when combining with the information from the inner detectors. We note here in the text we will not distinguish tracks and shower when discussing HGCAL, since the shower can be viewed as a "fat track". In general, the energy deposit pattern in EM calorimeter for photons, electrons and positrons are indistinguishable. Since the EM calorimeter of HGCAL has integrated 28 tracking layers, the resolution for electron and positron should be better. For charged hadrons like pions, the track extends from EM part to hadron part of HGCAL, which passes even more tracking layers. However, the hadronic tracks come in clusters and may degrade the performance. We assume that the angular resolution of the hadronic shower is the same as the EM shower. A subtle difference is HGCAL will be able to see neutral particles shower as well, which traditionally do not correspond to tracks.

\footnotetext{
${ }^{2}$ In principle, the secondary displacement from the heavy-light mesons, such as $B$ mesons and Kaons can help to identify the specific property of the LLPs.
} 
A schematic plot for the long-lived particle signal arriving the HGCAL is shown in figure 3. The particle will travel in a magnetic field of $B=3.8 \mathrm{~T}$ along the $z$ direction, therefore, it would follow a helical trajectory. We require the tracks to go through the first layer of HGCAL at $|z|=3.2 \mathrm{~m}$. The tracks with $p_{T}$ above $1 \mathrm{GeV}$ can be reconstructed at L1 level [79]. Each point on the track trajectory has a 4D coordinate, $(t, x, y, z)$. Once the momentum of a particle at a point on the track is known, the 4D trajectory of the full track can be calculated.

The directions of particles reaching HGCAL can be measured with an angular resolution of $\sigma_{\theta}$. The inaccuracy in measuring its direction is a main source of the error in the measurement of the track direction, which can fake our signal. We smear the direction of the momentum using a Gaussian function with a spread equal to the angular resolution $\sigma_{\theta}$. With this new momentum for the particle at the first layer of HGCAL, we then recalculate its $4 \mathrm{D}$ spiral trajectory.

\subsection{Signal and background generation}

\subsubsection{The long-lived particle signal}

The signal events at parton level are generated using MadGraph5_aMC@NLO [92], and the parton shower is performed by Pythia8 [93, 94]. The charged particles with $p_{T}>1 \mathrm{GeV}$ are kept as track candidates.

For the signal, the displaced tracks dominantly come from the displaced decay of the LLP $X$, which will give a displaced vertex (DV). The location and time of this DV results from a convolution of $X$ momentum distribution and the lifetime of $X$. We also require $X$ to decay within $|z|<1.5 \mathrm{~m}$ to ensure the tracks have five stubs in the tracker. Given the $4 \mathrm{D}$ vertex information and the 4-momentum of each charged particle at that vertex, one can reconstruct its $4 \mathrm{D}$ helical trajectory in the magnetic field. From this, we obtain the 3-momentum of the particle when it arrives at the HGCAL. We then smear the direction of its momentum and recalculate the $4 \mathrm{D}$ trajectory.

A further improvement of the HGCAL coverage can be achieved by considering LLPs decaying inside HGCAL. The LLP signal would appear as showers with an anomalous shape in the HGCAL. However, given the difficulty of modeling the showering pattern in this material-dense area and the lack of understanding of the background, we take the rather conservative class of signals in which $X$ decay before entering HGCAL. In this case, we use HGCAL to only pick out the displaced tracks. These tracks are identified via the showering of the hadronic particles from the LLP decay. Hence, they have a degraded angular resolution than the HGCAL physical limitations due to the broadening caused by interaction with materials. ${ }^{3}$ We also require these tracks to match hits in the outer part of the tracking system, which picks only the charged components of the signal. This is clearly a very conservative use of the HGCAL capability and leaves a large room for future improvement with a full understanding of the HGCAL performance.

\subsubsection{SM QCD background}

The main SM prompt backgrounds are the QCD dijet events, including bottom quark pair $b \bar{b}$. A main feature of the signals is the presence of tracks with large transverse impact

\footnotetext{
${ }^{3}$ We take this into account by using a degraded angular resolution.
} 
parameters. There are two reasons for such a QCD background to also have displaced tracks. The first one is the finite lifetime of mesons and baryons. The second one is from the finite angular resolution of HGCAL.

We use MadGraph5_aMC@NLO [92] and Pythia8 [93, 94] to generate the SM background events, which properly include the finite lifetime effect of SM mesons and baryons. The displaced tracks come primarily from $K_{S}^{0}$ meson $(c \tau \sim 2.7 \mathrm{~cm})$, with some addition contribution from heavy baryons like $\Lambda^{0}(c \tau \sim 7.8 \mathrm{~cm})$.

After applying generator level cuts such as $p_{T}>20 \mathrm{GeV}$ at the parton level, the crosssections of $b \bar{b}$ and $j j$ are $3.6 \times 10^{6} \mathrm{pb}$ and $1.7 \times 10^{8} \mathrm{pb},{ }^{4}$ respectively. The jet matching has been applied with one extra jet added and the minimal $k_{t}$ is set to be $30 \mathrm{GeV}$. After hadronization, charged tracks with $p_{T}>1 \mathrm{GeV}$ are kept. Among the tracks arriving at HGCAL, we kept the five leading ones to be smeared. ${ }^{5}$

\subsubsection{Fake-track background}

We denote as fake-track background the events with mis-reconstructed tracks from the accidental connections of the hits in the tracker system. They can easily have very large $d_{0}$, similar to those from the signal. There are $\mathcal{O}(30)$ such tracks per bunch crossing. This high combinatorics makes it possible for a selection of a few tracks to approximately form a vertex.

We follow refs. [95, 96] to generate events with mis-reconstructed tracks. We also add the timing information to the tracks, which can potentially further reduce the background $[97,98]$. To generate a fake-track, we use a set of kinematical variables following a flat distribution within the ranges indicated below, which was reproduced by CMS with a full simulation [99] and from the estimates of the expected occupancy of the trigger system [100].

- $\phi_{0} \in[0,2 \pi]$ : the azimuthal angle of a reference point from the beam spot in the $x-y$ plane.

- $z_{0} \in[-0.15,0.15] \mathrm{m}$ : the $z$ coordinate of the reference point.

- $t_{0} \in[-6,6]$ nanosecond: the time coordinate of the reference point.

- $d_{0} \in\left[10^{-3}, 0.15\right] \mathrm{m}$ : the transverse impact parameter of the track.

- $q / R \in\left[0,(1.75 \mathrm{~m})^{-1}\right]:$ the inverse of the track curvature in $x-y$ plane.

- $\eta \in[-3,3]$ : the pseudo-rapidity of the direction of the track at the reference point.

The reference point is defined at the location of the transverse impact parameter of a given track. The curvature of the track and the transverse momentum of the presumed particle responsible for it satisfy $R=\left|p_{T} /(q \times B)\right|=\left(p_{T} / \mathrm{GeV}\right) \times 0.88 \mathrm{~m} . q$ is the charge of the particle, assumed to be $\pm e$ with equal probability. From the range of the curvature, the

\footnotetext{
${ }^{4}$ Here we use the 4-flavor PDF scheme.

${ }^{5}$ This procedure tends to overestimate the suppression provided by our vertexing cuts. However, since our results essentially do not rely on the vertexing cuts for suppressing the SM QCD background, we keep only the five leading tracks for simplicity.
} 
tracks generated must have $p_{T} \geq 2 \mathrm{GeV}$, with a flat probability in $p_{T}^{-1}$. In the $x-y$ plane, the trajectory of the track is a circle with a radius equals to $R$. However, the origin (the beam spot) can be either inside or outside the circle. The distance between the center of the circle and the origin can be either $R-d_{0}$ or $R+d_{0}$. We assume the two cases occur with equal probability. With these parameters, the $4 \mathrm{D}$ trajectory of the fake tracks can be determined.

\section{$2.4 \quad$ Triggering strategy}

For the VBF channel, we require at least one forward jet $p_{T}>110 \mathrm{GeV}$, and both of the forward jets $p_{T}>35 \mathrm{GeV}$ with an invariant mass $m_{j j}>620 \mathrm{GeV}$ [101].

For the ggF channel, we try two different trigger strategies. First, we use a proposed L1 displaced track trigger cuts with $H_{T}>100 \mathrm{GeV}$, which has been demonstrated with two displaced tracks with $p_{T}>2 \mathrm{GeV}$ within an L1 jet [91]. This L1 trigger rate is about $10 \mathrm{kHz}$ in the central region and about a factor of 2-3 higher in the endcap region [91]. We require our signals to have more than five displaced tracks and $H_{T}>100 \mathrm{GeV}$, which is more stringent than ref. [91]. Nevertheless, we still assume the same level of L1 trigger rate of $10 \mathrm{kHz}$. Because that displaced track selection and vertex reconstruction do provide suppression of the L1 rate, the average number of multiple track bundles passing all these trigger requirements should be around one per triggered event. Given that the HL-LHC will run for $10^{8}$ seconds, the total number of such fake-track bundle events is about $1 \times 10^{12}$.

The second trigger strategy for the $\mathrm{ggF}$ channel is a displaced track trigger without the $H_{T}$ cut. It makes use of five displaced tracks with a vertex fitting, rather than the two displaced tracks [91]. This should reduce the low-level trigger rate and allow for the removal of the $H_{T}$ requirement. We also emphasize that these randomly connected tracks may not be corresponding calorimeter energy deposits in the HGCAL. Even if our estimate of the tracking alone suppression is not sufficient, consistency matching between different sub-detectors of the experiment will provide sufficient suppression.

\section{The kinematics of signal and backgrounds}

There are two main characteristics of the signal. First, the signal tracks tend to have large impact parameter, $d_{0}$. Hence, requiring a number of tracks (five in our case) to have large $d_{0}$ allows us to effectively separates the signal from the QCD background, which is mostly prompt. On the other hand, the fake-track background have a flat distribution in a large range of $d_{0}$. This is where the second main characteristic comes into play. Namely, the signal tracks all originate from a single vertex. Since each fake-track is independent of each other, they have a small probability of reconstructing a common vertex. In the following, we will define a set of variables to quantify this feature. We note that if the tracks are generated via interaction with detector material, there would be a reconstructable displaced vertex. One could veto all the displaced vertices in the materiel-dense region, as has been done by many LLP searches $[1,8,102]$.

\subsection{The displaced vertex fitting variables}

We fit the candidate tracks to a displaced vertex and define associated fitting variables as follows. We begin with five leading (in $p_{T}$ ) tracks and calculate their $4 \mathrm{D}$ trajectories. We 
perform a $2 \mathrm{D}$ vertex fit in the transverse plane by minimizing the following quantity,

$$
\Delta \mathrm{D} \equiv \sqrt{\sum_{i=1}^{5}\left(\sqrt{\left(x-x_{i}^{\mathrm{cen}}\right)^{2}+\left(y-y_{i}^{\mathrm{cen}}\right)^{2}}-R_{i}\right)^{2}},
$$

where $\left\{x_{i}^{\mathrm{cen}}, y_{i}^{\mathrm{cen}}\right\}$ are the $x-y$ coordinates of the center of the circle for the $i$ th track and $R_{i}$ is the transverse radius of the track helix. The minimization gives the best-fit coordinates , $x$ and $y$, for a presumed DV. Of course, this fit won't be perfect in reality and the tracks will miss the DV by some amount. To quantify this, we begin by identifying a point, with coordinate $\left(t_{i}, x_{i}, y_{i}, z_{i}\right)$, as the one corresponding to the DV on the $i$ th track. Since we have the full $4 \mathrm{D}$ trajectory of the track, we only need one parameter to identify this point. To this end, we choose to use the azimuth angle $\phi$ of the direction of the DV, with respect to the center of the circle of the $i$ th track. Comparing the $\phi$ change between the best fit DV $(x, y)$ and the hitting point at HGCAL layer, one can use the transverse velocity $v_{T}$ to fully determine $\left(t_{i}, x_{i}, y_{i}, z_{i}\right)$ for the DV on this particular track. Of course, in the ideal case in which all tracks originating from a DV are perfectly reconstructed, all of the $x_{i}$ and $y_{i}$ will coincide with $x$ and $y$. We can define the following variables associated with a fitted DV.

- The displacement of the vertex in the transverse plane $\mathrm{r}_{\mathrm{DV}} \equiv \sqrt{x^{2}+y^{2}}$ that minimizes $\Delta \mathrm{D}$ in eq. 3.1.

- The imperfectness or the spread of vertex fitting, $\Delta \mathrm{D}_{\min }$, based on the best-fit $2 \mathrm{D}$ vertex coordinates $x$ and $y$ that minimizes $\Delta \mathrm{D}$ in eq. 3.1.

- Based on the set of $\left\{z_{i}, t_{i}\right\}$ for each track that form a DV, we can define the mean value $\bar{z}$ and $\bar{t}$, and their standard deviations $\sigma_{\mathrm{z}}$ and $\sigma_{\mathrm{t}}$.

- For the $i$ th track, we define the time delay as $\Delta t_{i} \equiv t_{i}-\sqrt{x_{i}^{2}+y_{i}^{2}+z_{i}^{2}} / c$. We define the time delay of the displaced vertex $(\overline{\Delta t})$ as the average of the $\Delta t_{i}$ of the five leading tracks (in $p_{T}$ ) and the standard deviation $\sigma_{\Delta \mathrm{t}}$. For a slow-moving LLP which decays at the DV, $\Delta t$ would be its time delay in comparison to the prompt particles propagating from the interaction point to the DV.

In summary, we can define the following kinematic variables using the above 2D-4D displaced vertex fitting procedure,

$$
\mathrm{r}_{\mathrm{DV}}, \Delta \mathrm{D}_{\min }, \overline{\mathrm{t}}, \overline{\mathrm{z}}, \overline{\Delta \mathrm{t}}, \sigma_{\mathrm{t}}, \sigma_{\mathrm{z}}, \sigma_{\Delta \mathrm{t}} .
$$

In figure 4, we illustrate the fitted DV location in the $x-y$ plane and the five leading tracks in an event from the fake-track background, the QCD background, and the LLP signal. For the backgrounds, shown in (a), (b), and (c), we use solid red (dashed gray) lines for the trajectories after (before) the fitted DV. For the signal, shown in (d) and (e), we use solid red (dashed gray) lines for their trajectories after (before, extrapolated) the LLP decay. The fitted DV location is represented by a blue dot. The black circles have a radius representing the fitted vertex spread, $\Delta \mathrm{D}_{\text {min }}$. A smaller black circle indicates the 


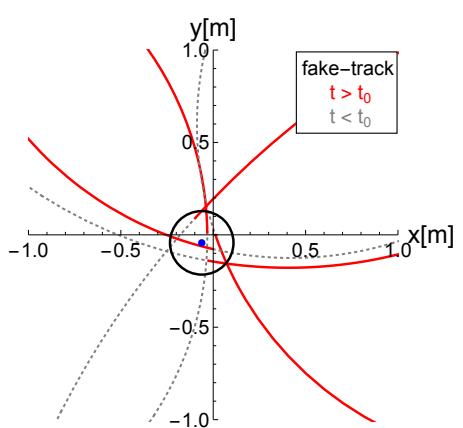

(a)

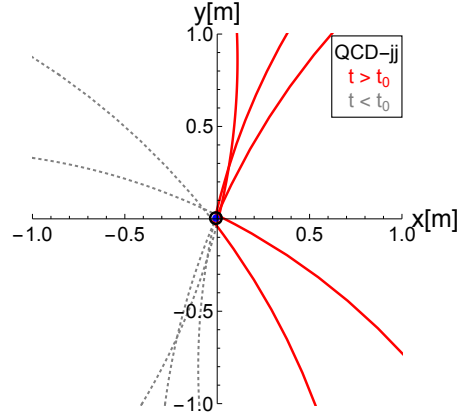

(b)

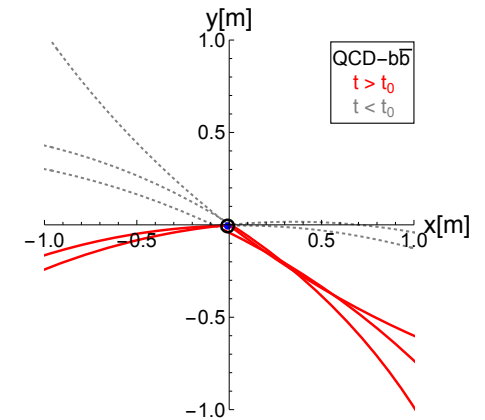

(c)

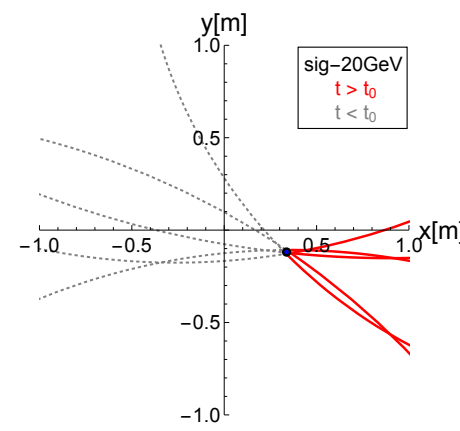

(d)

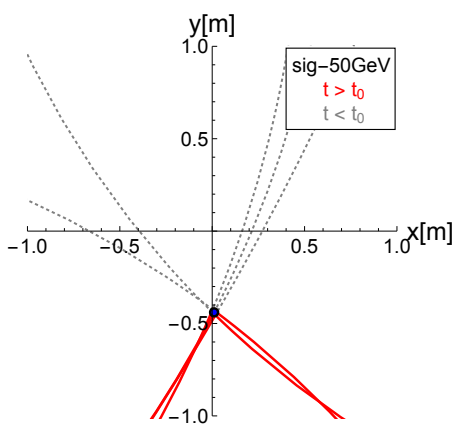

(e)

Figure 4. Illustrative event displays in the $x-y$ plane for the fitting algorithm with leading five displaced tracks. The blue dot is the fitted DV. The solid red (dashed gray) lines are the charged track trajectories in the event, after (before) the fitted DV. From left to right, the plots are for (a) fake-track background, (b) SM QCD light jet background, (c) SM QCD heavy-flavor jet background, (d) LLP signal with $m_{X}=20 \mathrm{GeV}$ and (e) LLP signal with $m_{X}=50 \mathrm{GeV}$.

vertex fitting algorithm successfully identifies the location of the displaced vertex. This figure shows the different behavior of the various types of background and the signal. For the SM QCD background, the vertices have small displacement, and the fitted vertex has sizable spread. For the fake-track background, the vertices can have large displacement, and the fitted vertices have a much larger spread, since the tracks are not correlated. For the signals, the fitted vertices would have small spread. For a lighter LLP (hence more boosted), shown in panel (d), the resulting tracks would be more collimated. For a heavier LLP, the resulting tracks spread like two sub-jets, as shown in panel (e).

The distribution of kinetic variables in eq. 3.2 are shown in figure 5 for the signal, QCD background and fake-track background. For the signal, we show two examples with $m_{X}=20$ and $50 \mathrm{GeV}$, with a lifetime $c \tau_{X}=1 \mathrm{~m}$. To better understand the effect of angular resolution, we show the distribution of variables without the angular smearing effect in figure 7 in appendix A. In general, since the fake tracks are randomly generated with a large spread in track parameters, we do not expect the angular resolution effect to change fake-track background significantly, which can be clearly seen in figure 5 and figure 7. Next, we explain the distribution for each variable in detail. 
(a)

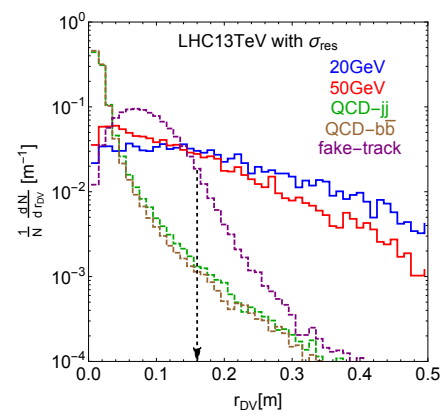

(b)

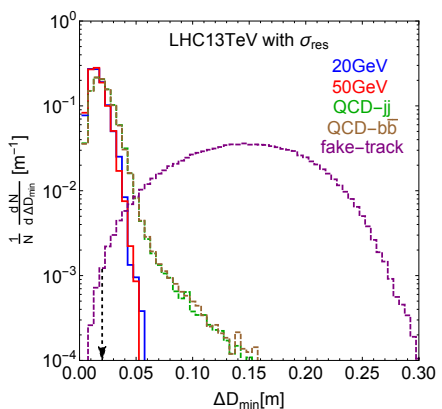

(c)

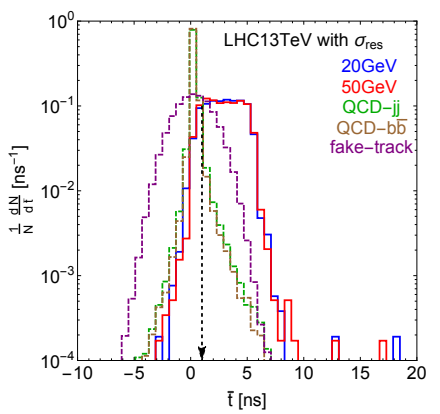

(f)

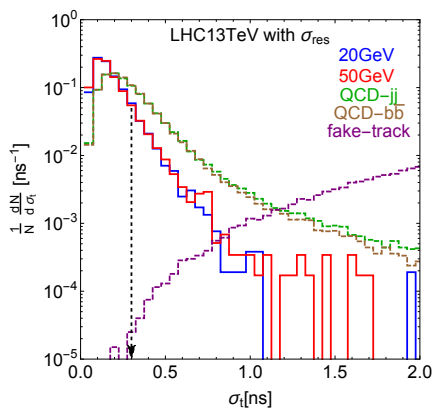

(d)

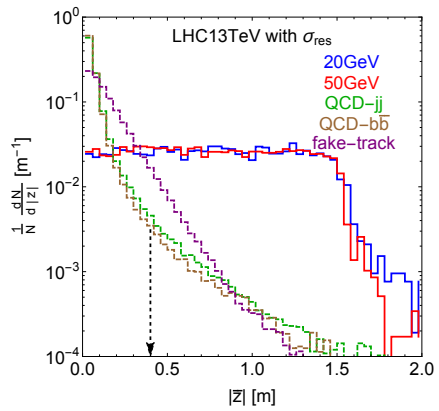

$(\mathrm{g})$

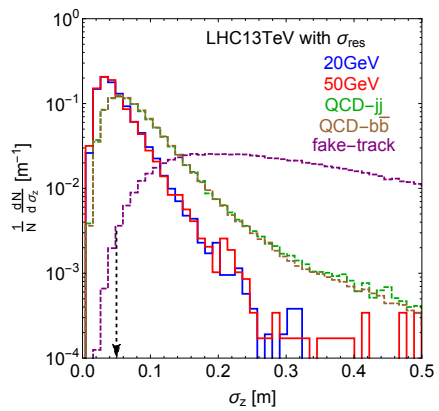

(e)

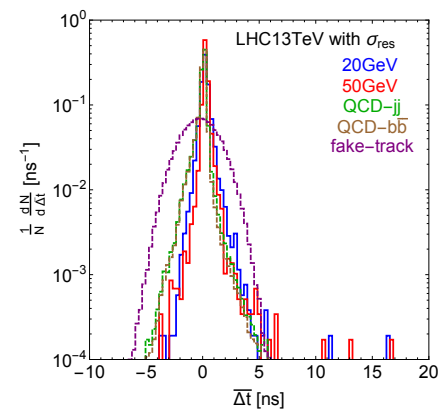

(h)

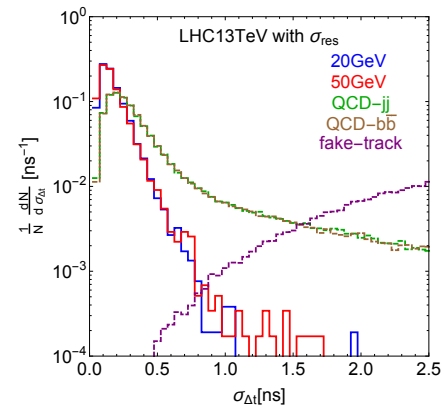

Figure 5. The kinetic distributions of the leading five tracks for the QCD background, fake-track background and the gluon fusion signal with the angular resolution effect applied. The variables are $\mathrm{r}_{\mathrm{DV}}, \Delta \mathrm{D}_{\text {min }}$ in the top row, $\overline{\mathrm{t}},|\overline{\mathrm{z}}|, \overline{\Delta \mathrm{t}}$ in the middle, and $\sigma_{\mathrm{t}}, \sigma_{\mathrm{z}}, \sigma_{\Delta \mathrm{t}}$ in the bottom row. For the signal, we take $m_{X}$ to be 20 and $50 \mathrm{GeV}$ respectively, with the same lifetime of $c \tau_{X}=1 \mathrm{~m}$. The vertical dotted black line indicates the cut proposed on the variables.

- $\mathrm{r}_{\mathrm{DV}}$ : the distance between DV and origin in the transverse plane.

The QCD backgrounds $j j$ and $b \bar{b}$ peak around zero, which means the fitted DV locates near the origin in the $x-y$ plane as most of the tracks from QCD are prompt. The distribution of $\mathrm{r}_{\mathrm{DV}}$ extends up to $\sim 0.3 \mathrm{~m}$. Comparing the results with (figure 5 ) and without (figure 7) $\sigma_{\theta}$, we see that the angular resolution does lead to a broader shape. However, turning off angular resolution does not lead to exact $\mathrm{r}_{\mathrm{DV}}=0 \mathrm{~m}$. Some charged tracks start from displaced vertexes from long lifetime mesons decay, 
e.g., $K_{S}^{0}$. There is no significant difference between QCD backgrounds $j j$ and $b \bar{b}$. The reason is that B-meson has a proper lifetime of $\sim 0.045 \mathrm{~cm}$, which is too small to generate a difference between $j j$ and $b \bar{b}$.

The fake-track background peaks at around $0.1 \mathrm{~m}$. The related variable from faketrack generation is $d_{0}$, which have a typical value of $0.1 \mathrm{~m}$. The fitted DV is not too far from the reference point for each track, because the reference point is the closest point on the track to the beam spot. This feature can be seen in figure 4 (a) as well. For the signal, $\mathrm{r}_{\mathrm{DV}}$ is approximately the position where $X$ particle decays in the $x-y$ plane. Its distribution has a very long tail, due to the lifetime of $X$ particle.

- $\Delta \mathrm{D}_{\min }$ : a measure of how well the set of candidate tracks fit in a common vertex.

Both QCD background and the signal should have a distribution of $\Delta \mathrm{D}_{\text {min }}$ peaks near zero. As shown in figure 5 (b), both of them have a similar shape and a spread of about $0.05 \mathrm{~m}$, mostly from the angular resolution of HGCAL. The size of $\Delta \mathrm{D}_{\text {min }}$ can be estimated as $\sim \sigma_{\theta} R \sqrt{5}=0.03 \mathrm{~m}$, with $R \simeq 3 \mathrm{~m}$, and the factor $\sqrt{5}$ comes from the sum of five tracks. This is consistent with figure $5(\mathrm{~b}) . \Delta \mathrm{D}_{\min }$ of the fake tracks peaks around $0.2 \mathrm{~m}$, since the tracks have a spread in $d_{0}$ of $\mathcal{O}(0.1 \mathrm{~m})$ and they do not fit well into a common vertex.

Turning off the angular resolution in figure 7 (b), the signal events all have exactly $\Delta \mathrm{D}_{\text {min }}=0 \mathrm{~m}$, which also shows that our algorithm correctly finds the DV where $X$ decays. For the QCD distributions, there are still a few percent of events with non-zero $\Delta \mathrm{D}_{\min }$, due to long-lived SM hadrons.

- $\bar{t}$ : the average of the time coordinate of the tracks at the fitted DV.

The QCD background peaks around zero as shown in figure 7 (c). The spread of $\bar{t}$ dominantly comes from the angular resolution, and it can be estimated to be $\Delta \phi R / v_{T} / \sqrt{5}$, where $v_{T}$ is the transverse velocity of the particle responsible for the track and $\Delta \phi$ is the azimuthal angle change when the track evolved from the fitted DV to the HGCAL. The geometrical acceptance of the HGCAL selects forward tracks, leading to smaller $v_{T} \sim 0.2 c$, as shown in figure 8 in the appendix. For $\Delta \phi$, its $1 \sigma$ spread is about 0.02 in figure 9 in the appendix. Therefore, for a typical track radius of $R=3 \mathrm{~m}$, the spread of $\bar{t}$ for QCD background is about $0.5 \mathrm{~ns}$, agreeing with figure 5 .

For the signal, $\bar{t}$ peaks around a few ns, due to the delayed decay of $X$. In both figure 5 and 7 , we have chosen $c \tau_{X}=1 \mathrm{~m}$ which corresponds to $3 \mathrm{~ns}$. Moreover, decay products from a lighter LLP (hence with a larger boost) has a larger $\bar{t}$ than that of a heavier LLP.

For the fake tracks, the $t_{i}$ for each track should be determined mainly by the random seed time $t_{0}$, ranging from $\{-6,6\}$ ns with a flat distribution. The distribution can be approximated by a Gaussian function peaking around zero, with a standard deviation of $3.5 / \sqrt{5}=1.6 \mathrm{~ns}$, as shown in figure 5 (c). Here 3.5 is an ad hoc standard deviation of the flat distribution of each track. In the limit of a large number of tracks, Gaussian 
function can be used to estimate the spread of the fitted vertex. From figure 7 (c), we see that the distribution from the fake-track background is not affected by angular resolution, as expected.

- $\sigma_{\mathrm{t}}$ : the standard deviation of the time-coordinates of the constituent tracks at the fitted DV.

For the signal and QCD background, the distribution is expected to be concentrated at small values, as shown in figure 7 . The spread dominantly comes from the angular resolution, as shown in figure 5 (f). The spread can be estimated by $\Delta \phi R / v_{T}$. As explained for $\bar{t}$, it is $\sim 1 \mathrm{~ns}$ for QCD background, which agrees with the broad distribution up to a few ns. In addition, some QCD events have large separation between the displaced tracks of the long-lived mesons and the prompt tracks. For the fake-track background, the spread is largely due to the uncorrelated large spread of the track seed time $t_{0}$ distributions.

- $\bar{z}$ : the averaged z-coordinate of the tracks at the fitted DV.

We first look at the distribution without $\sigma_{\theta}$ in figure 7 . The signals have a very flat distribution because of the long lifetime of $X$. There is a hard cut because $X$ is required to decay in the region $|z|<1.5 \mathrm{~m}$ to ensure five stubs for the signal track.

The finite angular resolution $\sigma_{\theta}$ effects on the distributions of the signal and QCD background are shown in figure 5 . The signal changes very little because the lifetime and the limited decay region are the dominant factors. The distribution of the QCD background is broadened in a similar fashion as its $\bar{t}$ distribution, the $1 \sigma$ spread is roughly $0.5 \mathrm{~ns} \times v_{z} \sim 0.15 \mathrm{~m}$ with $\left|v_{z}\right| \sim c$ as shown in figure 8 . The tail in the QCD background extends up to $\sim 2 \mathrm{~m}$ with less than $10^{-4}$ probability, in agreement with the $\bar{t}$ distribution which extends to around $\sim 8$ ns with similar probability. Since $8 \mathrm{~ns} \times c \sim 2.4 \mathrm{~m}$, this shows a correlation between $\bar{t}$ and $\bar{z}$. The distribution of the fake-track background follows the exponential shape $e^{-|z| / \sigma}$ with a spread of $\sim 0.15 \mathrm{~m}$.

- $\sigma_{\mathrm{z}}$ : the standard deviation of the z-coordinates of tracks from the fitted DV.

Starting with figure 7 without $\sigma_{\theta}$, it is exactly zero for the signal and almost zero for QCD background for the similar reason as $\sigma_{\mathrm{t}} . \sigma_{\theta}$ broadens the distributions up to $0.15 \mathrm{~m}$ for the signal and QCD background, which is in agreement with the previous estimate $\left(\Delta \phi R / v_{T}\right) v_{z} \sim 0.15 \mathrm{~m}$. The QCD background has a larger spread than signal, for the same reason as $\sigma_{\mathrm{t}}$. For the fake-track background, the large spread in the seed $z_{0}$ of the constituent tracks leads to a large spread.

- $\overline{\Delta t}$ : the average of the time delay for the tracks.

In figure $7, \Delta t$ of the signal comes from the slow-moving LLP $X$. Thus, the values of $\Delta t_{i}$ are always positive. Moreover, a heavier $X$ moves slower than a lighter $X$, thus the tail of heavier $X$ is longer than that of the lighter $X$ and the QCD background. The QCD background has a peak around 0 since the track is prompt. The spread 
around 0 is due to smearing effects and the fact that some tracks come from long-lived meson. The fake-track background distribution is Gaussian-like with 1- $\sigma$ spread of about $1.5 \mathrm{~ns}$. It is almost symmetric around zero since its $4 \mathrm{D}$ parameters are random and independent from each other. The largest spread comes from random $t_{i}$, thus $\overline{\Delta t}$ is very similar to $\bar{t}$.

- $\sigma_{\Delta t}$ : the standard deviation of the time delay of the tracks.

Starting with figure 7 without $\sigma_{\theta}$, the signal has exactly $\sigma_{\Delta t}=0$, while the QCD background peaks at zero with a spread from long-lived meson decay. The fake background is similar as in $\sigma_{\mathrm{t}}$ because the dominant spread comes from random $t_{0}$. After including $\sigma_{\theta}$, the distributions are broadened as expected but without qualitative change.

We see that the distributions of fake tracks are quite different from signal in general. Based on this, we propose the six cuts according to the distributions and the cut flow table is given in table 5. Explicitly, the cuts for DV fitting variables are,

$$
\mathrm{r}_{\mathrm{DV}}>0.16 \mathrm{~m}, \Delta \mathrm{D}_{\min }<0.02 \mathrm{~m}, \overline{\mathrm{t}}>1 \mathrm{~ns}, \sigma_{\mathrm{t}}<0.3 \mathrm{~ns}, \quad|\overline{\mathrm{z}}|>0.4 \mathrm{~m}, \sigma_{\mathrm{z}}<0.05 \mathrm{~m},
$$

which we denoted them collectively as vertexing-cuts.

\subsection{The transverse impact parameter distribution}

The $d_{0}$ distributions of the five tracks for the QCD background, fake-track background, and the signal are given in figure 6. In figure 6 (a), the magnetic field is set as zero, and the angular resolution effect is not included either. The fake tracks have a flat $d_{0}$ distribution from its definition. The signal has a broad distribution due to the delayed decay of $X$. Moreover, the lighter $X$ has a slightly narrower distribution since its decay products are more boosted. The QCD dijet background peaks at $d_{0}=0 \mathrm{~m}$, with a tail from the long-lived hadron decay.

In figure 6 (b), the effect of the magnetic field is included. Comparing with figure 6 (a), the QCD background from long-lived hadron are broadened, while the signal is less affected since the displacement before the $X$ decay is more important. The fake-track background is almost flat in $d_{0}$ by definition.

Both the magnetic field and the angular resolution effects are included in figure 6 (c). Comparing with figure 6 (b), the signal is almost unchanged. The QCD background is broadened with a spread of $0.015 \mathrm{~m}$. The spread can be estimated by $\sigma_{\theta}|z| \sim 0.015 \mathrm{~m}$, where $|z|$ is taken to be $3.2 \mathrm{~m}$, the distance to HGCAL. The fake-track background is still flat, with its edge smeared by the angular resolution.

In figure $6(\mathrm{~d})$, we have included both the magnetic field and angular resolution effect after applying vertexing-cuts. Importantly, the distribution of the QCD background is trimmed to be a Gaussian shape. This is expected since the outliers with large $d_{0}$ come from the decay of long-lived hadron, which fails the DV fitting (and thus fail to pass the vertexing-cuts). Comparing panel (c) and panel (d), we can see that the vertexing-cuts improve the per-track QCD background rejection power from $5 \times 10^{-2}$ to $<10^{-3}$ level. For 
(a)

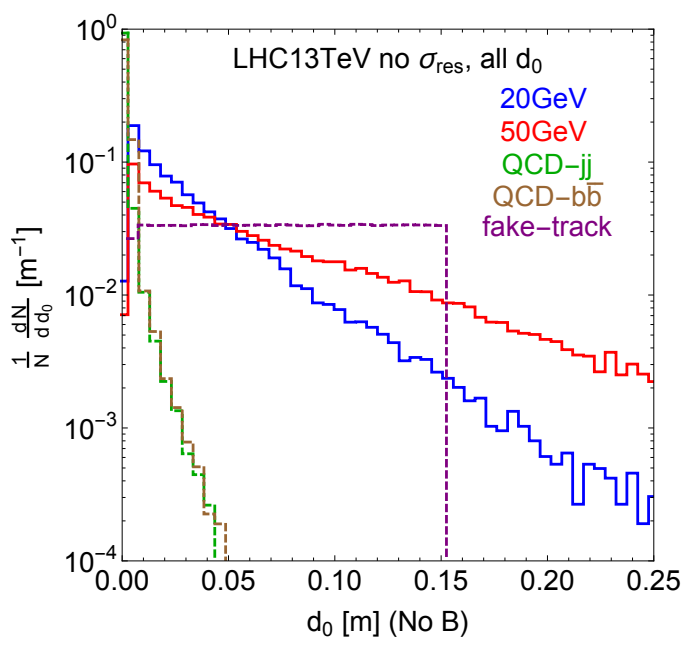

(c)

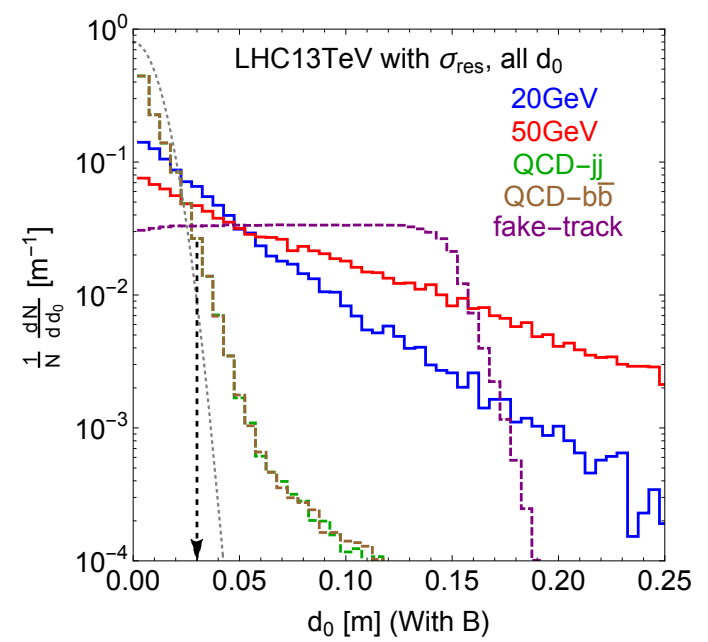

(b)

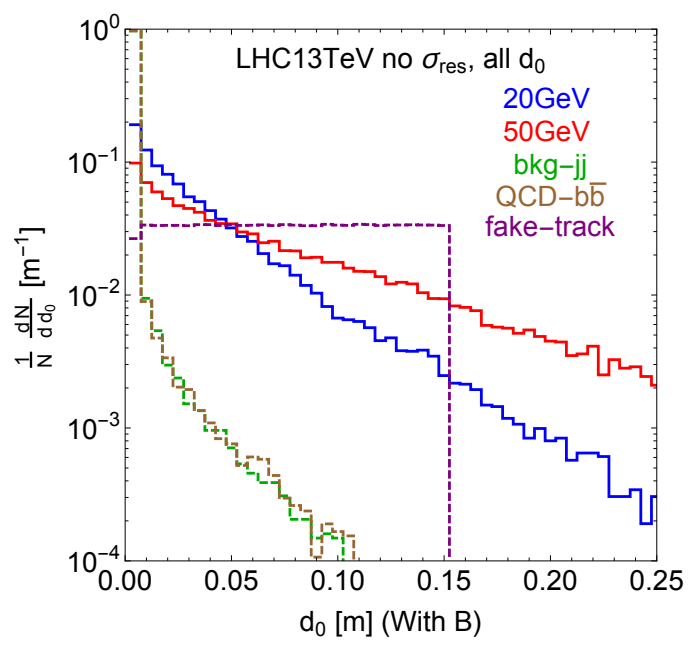

(d)

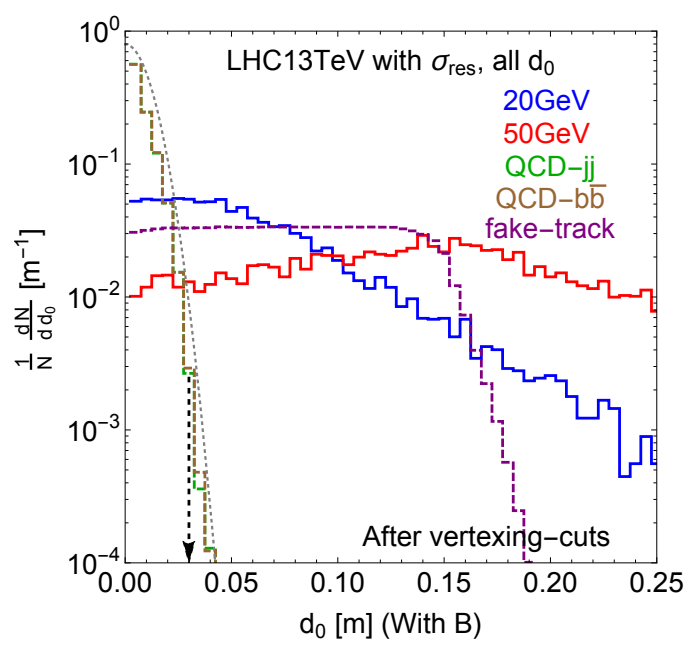

Figure 6. The distributions of the transverse impact parameter $d_{0}$ for the QCD background, the fake-track background and the signal. Panel (a) has no angular resolution effect and no magnetic field. Panel (b) has no angular resolution effect but with a magnetic field of 3.8 T. Panel (c) has the angular resolution effect and the magnetic field. Panel (d) has both effects, and with vertexing-cuts imposed. The dotted gray lines are the Gaussian function with a spread of $0.015 \mathrm{~m}$, corresponding to the angular resolution times the $z$ coordinate of the HGCAL.

the signal, the vertex cuts $\mathrm{r}_{\mathrm{DV}}>0.16 \mathrm{~m}, \overline{\mathrm{t}}>1 \mathrm{~ns}$, and $|\overline{\mathrm{z}}|>0.4 \mathrm{~m}$, selects events in which the $X$ particle decays far from the origin. Any track with small $d_{0}$ is significantly affected. The vertexing-cuts do not affect the distribution of $d_{0}$ of the fake-track background.

\subsection{Correlations between the selection cuts}

Due to the limited statistics of our simulation in some cases, we estimate cut efficiencies by the product of efficiencies of different subsets of cuts. To validate this approach, we study the correlations between those cuts. 


\begin{tabular}{|c|c|c|c|c|c|c|}
\hline$j j$ dijets & $\mathrm{r}_{\mathrm{DV}}>0.16 \mathrm{~m}(*)$ & $\Delta \mathrm{D}_{\min }<0.02 \mathrm{~m}$ & $\bar{t}>1 \mathrm{~ns}(*)$ & $\sigma_{\mathrm{t}}<0.3 \mathrm{~ns}$ & $|\overline{\mathrm{z}}|>0.4 \mathrm{~m}(*)$ & $\sigma_{\mathrm{z}}<0.05 \mathrm{~m}$ \\
\hline$\left(d_{0}>0.01 \mathrm{~m}\right)^{1}$ & 0.70 & 1.3 & 0.78 & 1.1 & 0.77 & 1.2 \\
\hline$\left(d_{0}>0.03 \mathrm{~m}\right)^{1}$ & 0.25 & 8.6 & 0.37 & 1.4 & 0.40 & 1.8 \\
\hline$\left(d_{0}>0.05 \mathrm{~m}\right)^{1}$ & 0.09 & 35.0 & 0.18 & 2.4 & 0.19 & 3.2 \\
\hline$b \bar{b}$ dijets & $\mathrm{r}_{\mathrm{DV}}>0.16 \mathrm{~m}(*)$ & $\Delta \mathrm{D}_{\min }<0.02 \mathrm{~m}$ & $\bar{t}>1 \mathrm{~ns}(*)$ & $\sigma_{\mathrm{t}}<0.3 \mathrm{~ns}$ & $|\overline{\mathrm{z}}|>0.4 \mathrm{~m}(*)$ & $\sigma_{\mathrm{z}}<0.05 \mathrm{~m}$ \\
\hline$\left(d_{0}>0.01 \mathrm{~m}\right)^{1}$ & 0.71 & 1.3 & 0.78 & 1.1 & 0.77 & 1.2 \\
\hline$\left(d_{0}>0.03 \mathrm{~m}\right)^{1}$ & 0.21 & 8.8 & 0.36 & 1.4 & 0.36 & 1.8 \\
\hline$\left(d_{0}>0.05 \mathrm{~m}\right)^{1}$ & 0.07 & 47.0 & 0.16 & 2.6 & 0.17 & 4.0 \\
\hline
\end{tabular}

Table 1. The correlations $\rho$ (vertexing-cuts, $d_{0}$ ) for QCD $j j$ and $b \bar{b}$ backgrounds. The columns with $(*)$ are not used to calculate the final selection efficiencies.

To quantify the correlations among different cuts, we use the following function

$$
\rho_{A, B} \equiv \frac{\epsilon(A) \epsilon(B)}{\epsilon(A \& B)}
$$

where $A$ and $B$ are different cut variables. $\epsilon(A)$ is the efficiency for imposing the corresponding cut $A$, while $\epsilon(A \& B)$ is the cut efficiency when both $A$ and $B$ cuts are applied. $\rho_{A, B}=1$ means $A$ and $B$ cuts are completely independent. If $\rho_{A, B} \approx \mathcal{O}(1), \mathrm{A}$ and $\mathrm{B}$ are approximate independent. When $\rho_{A, B} \ll 1, \epsilon(A) \epsilon(B)$ underestimates $\epsilon(A \& B)$. In this case, using $\epsilon(A) \epsilon(B)$ is inappropriate for selection efficiency estimation. For $\rho_{A, B} \gg 1, \epsilon(A) \epsilon(B)$ is a conservative estimate for background. In summary, if $\rho \gtrsim 1$, using the product of the individual cuts is a reasonable estimate.

We begin with the QCD $j j$ and $b \bar{b}$ backgrounds. First of all, the correlations among vertexing-cuts variables are not needed, since we have enough simulated events to compute the efficiency without relying using the product of efficiencies of the individual cuts. However, the vertexing-cuts are not enough to suppress the background; we further require multiple tracks with large $d_{0}$. Here, we are limited by the statistics. Hence, we need to check the correlation between vertexing-cuts and $d_{0}$ cuts, and the correlation among different $d_{0}$ cuts.

The correlations between vertexing-cuts and the $d_{0}$ cut are given in table 1 . With higher cut threshold of $d_{0}$, the correlation between single $d_{0}$ cut and $\mathrm{r}_{\mathrm{DV}}, \overline{\mathrm{t}}$ and $|\overline{\mathrm{z}}|$ becomes stronger. This is expected. For $j j$ and $b \bar{b}$ QCD backgrounds, the event with large transverse impact parameter is also likely to have large values for $\mathrm{r}_{\mathrm{DV}}, \bar{t}$ and $|\bar{z}|$. Therefore, we will not use these cuts when calculating the final selection efficiencies. In this way, we avoid double counting and remain conservative because all the remaining columns have $\rho>1$.

Next, we would estimate the cut efficiency on the QCD dijet background by the product of single track efficiency of $d_{0}>0.03 \mathrm{~m}$. We would like to show that the consecutive $d_{0}$ cuts are approximately independent. This is expected since the large $d_{0}$ tracks are mainly from detector resolution effects, which are independent between tracks. As shown in figure 10 in the appendix, the $d_{0}$ distribution of the leading track is the same as the ensemble of the five tracks shown in figure 6 . This indicates that we could apply the transverse impact 


\begin{tabular}{|c|c|c|c|c|c|}
\hline$j j$ dijets & $d_{0}>0.01 \mathrm{~m}$ & $d_{0}>0.015 \mathrm{~m}$ & $d_{0}>0.02 \mathrm{~m}$ & $d_{0}>0.025 \mathrm{~m}$ & $d_{0}>0.03 \mathrm{~m}$ \\
\hline$\rho_{d}^{1}$ & $0.970 \pm 0.016$ & $0.990 \pm 0.027$ & $1.000 \pm 0.056$ & $1.10 \pm 0.15$ & $1.40 \pm 0.45$ \\
\hline$\rho_{d}^{2}$ & $1.20 \pm 0.04$ & $1.20 \pm 0.10$ & $0.69 \pm 0.17$ & - & - \\
\hline$\rho_{d}^{3}$ & $1.30 \pm 0.11$ & $1.20 \pm 0.35$ & - & - & - \\
\hline$\rho_{d}^{4}$ & $1.60 \pm 0.30$ & - & - & - & - \\
\hline$\rho_{d}^{5}$ & $1.80 \pm 0.83$ & - & - & - & - \\
\hline$b \bar{b}$ dijets & $d_{0}>0.01 \mathrm{~m}$ & $d_{0}>0.015 \mathrm{~m}$ & $d_{0}>0.02 \mathrm{~m}$ & $d_{0}>0.025 \mathrm{~m}$ & $d_{0}>0.03 \mathrm{~m}$ \\
\hline$\rho_{d}^{1}$ & $1.000 \pm 0.017$ & $1.000 \pm 0.029$ & $1.000 \pm 0.054$ & $1.30 \pm 0.17$ & $1.80 \pm 0.60$ \\
\hline$\rho_{d}^{2}$ & $1.100 \pm 0.041$ & $1.10 \pm 0.09$ & $1.00 \pm 0.29$ & - & - \\
\hline$\rho_{d}^{3}$ & $1.100 \pm 0.087$ & $0.84 \pm 0.22$ & - & - & - \\
\hline$\rho_{d}^{4}$ & $1.00 \pm 0.15$ & - & - & - & - \\
\hline$\rho_{d}^{5}$ & $0.62 \pm 0.16$ & - & - & - & - \\
\hline
\end{tabular}

Table 2. The correlation (including the statistical uncertainty) from our simulation, for multiple $d_{0}$ tracks for QCD dijet backgrounds after applying vertexing-cuts. For the entries with "_", there are not enough statistics to make a reliable estimate. For higher tracks multiplicity and $d_{0}$ threshold, the results suffer from larger fluctuations due to limited statistics.

parameter cut on multiple tracks independently. ${ }^{6}$ To quantify this further, we define the following function to study the correlations between different $d_{0}$ cuts,

$$
\rho_{d}^{n} \equiv \frac{\epsilon^{n}\left(1 \text { track } d_{0}>0.03 \mathrm{~m}\right)}{\epsilon\left(\mathrm{n} \text { tracks } d_{0}>0.03 \mathrm{~m}\right)}
$$

where $d_{0}>0.03 \mathrm{~m}$ is chosen as an example. Note the tracks in numerator are randomly chosen, while in the denominator they are the $n$ hardest tracks. The correlation $\rho_{d_{n}}$ for QCD $j j$ and $b \bar{b}$ backgrounds after imposing vertexing-cuts are given in table 2 .

In table 2 , from $\rho_{d}^{1}$ to $\rho_{d}^{5}$, the correlations are mostly around 1 , implying the $d_{0}$ cuts for different tracks are indeed independent. ${ }^{7}$ After applying the vertexing-cuts and requiring $d_{0}>0.03 \mathrm{~m}$, we are again limited by the statistics of our simulation. For this reason, $\rho_{d}^{1}$ deviates significantly from 1 here. Similarly, for the entries with "-", there are not enough statistics to make a reliable estimate. We also check the correlation for multiple $d_{0}$ tracks without applying vertexing-cuts. The results are shown in table 6 in the appendix. As expected, they are approximately independent.

Next, we discuss the correlations of the cuts for the fake-track background. Firstly, among the vertexing-cuts variables, table 3 shows that most of them are close to 1 , which means approximately independent. Two of the correlations are much larger than 1, which means using the product of the individual cuts efficiency is a reasonable estimate. However,

\footnotetext{
${ }^{6}$ This independence of the tracks is true for both prompt QCD background from smearing effects and for the fake-track background. For the displaced tracks from long-lived hadrons, there is a certain level of correlations which is already removed by our vertexing-cuts. Hence, we ignore these minor correlations here.

${ }^{7} \rho_{d}^{1}$ is not exactly 1 , because the track in the numerator is randomly picked, while the track in the denominator is the leading track. Thus, the fact that its value is close to 1 is a kind of proof that different tracks are independent.
} 


\begin{tabular}{|c|c|c|c|c|c|c|}
\hline fake tracks & $\mathrm{r}_{\mathrm{DV}}>0.16 \mathrm{~m}$ & $\Delta \mathrm{D}_{\min }<0.02 \mathrm{~m}$ & $\bar{t}>1 \mathrm{~ns}$ & $\sigma_{\mathrm{t}}<0.3 \mathrm{~ns}$ & $|\overline{\mathrm{z}}|>0.4 \mathrm{~m}$ & $\sigma_{\mathrm{z}}<0.05 \mathrm{~m}$ \\
\hline $\mathrm{r}_{\mathrm{DV}}>0.16 \mathrm{~m}$ & & $0.49 \pm 0.04$ & $0.85 \pm 0.01$ & $2.49 \pm 2.51$ & $0.156 \pm 0.001$ & $216.0 \pm 216.0$ \\
\hline$\Delta \mathrm{D}_{\min }<0.02 \mathrm{~m}$ & $0.49 \pm 0.04$ & & $0.95 \pm 0.04$ & - & $0.62 \pm 0.04$ & $2.12 \pm 0.95$ \\
\hline $\bar{t}>1 \mathrm{~ns}$ & $0.85 \pm 0.01$ & $0.95 \pm 0.04$ & & $0.69 \pm 0.17$ & $0.80 \pm 0.01$ & $1.05 \pm 0.03$ \\
\hline$\sigma_{\mathrm{t}}<0.3 \mathrm{~ns}$ & $2.49 \pm 2.51$ & - & $0.69 \pm 0.17$ & & $0.87 \pm 0.45$ & $0.25 \pm 0.25$ \\
\hline$|\overline{\mathrm{z}}|>0.4 \mathrm{~m}$ & $0.156 \pm 0.001$ & $0.62 \pm 0.04$ & $0.80 \pm 0.01$ & $0.87 \pm 0.45$ & & $18.86 \pm 4.72$ \\
\hline$\sigma_{\mathrm{z}}<0.05 \mathrm{~m}$ & $216.0 \pm 216.0$ & $2.12 \pm 0.95$ & $1.05 \pm 0.03$ & $0.25 \pm 0.25$ & $18.86 \pm 4.72$ & \\
\hline
\end{tabular}

Table 3. The correlation table for vertexing-cuts variables for the fake-track background. The entries with "_." contain too few events to make a reliable estimate and the statistical uncertainties are given after \pm .

\begin{tabular}{|c|c|c|c|c|c|c|}
\hline fake tracks & $\mathrm{r}_{\mathrm{DV}}>0.16 \mathrm{~m}$ & $\Delta \mathrm{D}_{\min }<0.02 \mathrm{~m}$ & $\bar{t}>1 \mathrm{~ns}$ & $\sigma_{\mathrm{t}}<0.3 \mathrm{~ns}$ & $|\bar{z}|>0.4 \mathrm{~m}$ & $\sigma_{\mathrm{z}}<0.05 \mathrm{~m}$ \\
\hline$\left(d_{0}>0.01 \mathrm{~m}\right)^{1}$ & 0.97 & 1.00 & 1.00 & 0.94 & 0.98 & 1.0 \\
\hline$\left(d_{0}>0.03 \mathrm{~m}\right)^{1}$ & 0.91 & 1.10 & 1.00 & 1.20 & 0.94 & 1.0 \\
\hline$\left(d_{0}>0.05 \mathrm{~m}\right)^{1}$ & 0.85 & 1.10 & 1.00 & 1.00 & 0.91 & 1.1 \\
\hline$\left(d_{0}>0.03 \mathrm{~m}\right)^{5}$ & 0.65 & 1.00 & 0.99 & 0.76 & 0.77 & 1.2 \\
\hline
\end{tabular}

Table 4. The correlation between vertexing-cuts and $d_{0}$ cuts for fake-track background.

both of them have large statistical errors. Moreover, due to limited statistics, there is no reliable estimate for the correlations between $\sigma_{\mathrm{t}}-\Delta \mathrm{D}_{\min }$. As a further check, we evaluated the correlations with a looser set of cuts thus containing more statistics, shown in table 7 in the appendix. For example, we relaxed the maximum $\sigma_{\mathrm{t}}$ cut to $0.5 \mathrm{~ns}$ rather than 0.3 ns. In this case, we see that all the variables are approximately independent. As a result, we conclude that the total cut efficiency for the vertexing-cuts variables estimated by using the product of the single cut efficiencies is reasonable.

We note that there is enough statistics in the fake-track background to calculate the efficiency of the multiple $\left(d_{0}>0.03 \mathrm{~m}\right)$ cuts without approximation. Hence, there is no need to check the correlations among individual $d_{0}$ cuts here. We are left to check the independence between vertexing-cuts and $d_{0}$ cuts, which is given in table. 4 . The first three rows show the correlations between the vertexing-cuts and various single $d_{0}$ cut from $0.01 \mathrm{~m}$ to $0.05 \mathrm{~m}$. In the fourth row, we use the exact $d_{0}$ cuts for five tracks. The result shows the correlations between vertexing-cuts and full $d_{0}$ cuts are approximate independent.

\section{The results}

\subsection{Cut efficiencies}

In this section, we present the efficiencies of cuts we adopt in this analysis in table $5 . N_{\text {ini }}$ is the initial event number from the cross-section only. $N_{\text {fin }}$ is final event numbers after imposing the trigger and the cuts in the table at the HL-LHC with $13 \mathrm{TeV}$ center-of-mass energy and $3 \mathrm{ab}^{-1}$ integrated luminosity. The row " 5 tracks" comes from the requirement that at least five tracks that arrive HGCAL and the trigger requirement. For the signal, it is 


\begin{tabular}{|c|c|c|c|c|c|}
\hline cut conditions & $j j$ dijet & $b \bar{b}$ dijet & fake-track & $g g F m_{s}=20 \mathrm{GeV}$ & $g g F m_{s}=50 \mathrm{GeV}$ \\
\hline$N_{\text {ini }}$ & $5.1 \times 10^{14}$ & $1.1 \times 10^{13}$ & $1 \times 10^{12}$ & $1.3 \times 10^{8} \times \mathrm{BR}$ & $1.3 \times 10^{8} \times \mathrm{BR}$ \\
\hline 5 tracks & $8.7 \times 10^{-1}$ & $8.4 \times 10^{-1}$ & 1.0 & $8.3 \times 10^{-2}$ & $2.1 \times 10^{-1}$ \\
\hline $\mathrm{r}_{\mathrm{DV}}>0.16 \mathrm{~m}$ & $9.2 \times 10^{-3}(*)$ & $7.5 \times 10^{-3}(*)$ & $4.5 \times 10^{-2}$ & $4.8 \times 10^{-1}$ & $3.1 \times 10^{-1}$ \\
\hline$\Delta \mathrm{D}_{\min }<0.02$ & $6.1 \times 10^{-1}$ & $6.1 \times 10^{-1}$ & $2.2 \times 10^{-3}$ & $8.7 \times 10^{-1}$ & $8.9 \times 10^{-1}$ \\
\hline $\bar{t}>1 \mathrm{~ns}$ & $3.3 \times 10^{-2}(*)$ & $2.8 \times 10^{-2}(*)$ & $2.8 \times 10^{-2}$ & $9.9 \times 10^{-1}$ & $9.9 \times 10^{-1}$ \\
\hline$\sigma_{\mathrm{t}}<0.3 \mathrm{~ns}$ & $7.1 \times 10^{-1}$ & $7.2 \times 10^{-1}$ & $4.5 \times 10^{-5}$ & $9.6 \times 10^{-1}$ & $9.8 \times 10^{-1}$ \\
\hline$|\bar{z}|>0.4 \mathrm{~m}$ & $3.4 \times 10^{-2}(*)$ & $2.8 \times 10^{-2}(*)$ & $6.4 \times 10^{-2}$ & $9.9 \times 10^{-1}$ & $9.9 \times 10^{-1}$ \\
\hline$\sigma_{\mathrm{z}}<0.05$ & $4.9 \times 10^{-1}$ & $4.9 \times 10^{-1}$ & $4.9 \times 10^{-3}$ & $8.5 \times 10^{-1}$ & $8.8 \times 10^{-1}$ \\
\hline$\epsilon_{\mathrm{vtc}}$ & $2.1 \times 10^{-1}$ & $2.1 \times 10^{-1}$ & $4.0 \times 10^{-13}$ & $3.4 \times 10^{-1}$ & $2.4 \times 10^{-1}$ \\
\hline$\left(d_{0}>0.03 \mathrm{~m}\right)^{5}$ & $\left(5.7 \times 10^{-4}\right)^{5}$ & $\left(6.8 \times 10^{-4}\right)^{5}$ & $3.4 \times 10^{-1}$ & $2.6 \times 10^{-1}$ & $8.1 \times 10^{-1}$ \\
\hline$N_{\text {fin }}$ & $5.7 \times 10^{-3}$ & $2.9 \times 10^{-4}$ & $1.4 \times 10^{-1}$ & $9.7 \times 10^{5} \times \mathrm{BR}$ & $5.3 \times 10^{6} \times \mathrm{BR}$ \\
\hline
\end{tabular}

Table 5. The cut-flow table for the QCD background, the fake-track background and the signal. $N_{\text {ini }}$ and $N_{\text {fin }}$ are the initial and final event numbers before and after imposing the cuts. These numbers correspond to an integrated luminosity of $\mathcal{L}=3 \mathrm{ab}^{-1}$ at the HL-LHC. "5 tracks" requires each track has $p_{T}>1 \mathrm{GeV}$ and at least 5 tracks arrive at HGCAL. " $\epsilon_{\mathrm{vtc}}$ " is the total efficiency for the vertexing-cuts except those with $(*)$. The efficiency of the $d_{0}$ cuts is calculated after applying the vertexing-cuts. We used the two signal benchmarks with $m_{X}=20$ and $50 \mathrm{GeV}$, and lifetime $c \tau_{X}=1 \mathrm{~m}$.

the combination of the geometric probability for $X$ decay inside the $|z|<1.5 \mathrm{~m}$ region and the efficiency for tracks arriving HGCAL. The QCD backgrounds have a better efficiency for tracks arriving HGCAL, because their tracks are more forward than the signal (see the upper panel of figure 8). Furthermore, the background jets are usually more energetic thus containing more tracks than the signal, which makes it much easier to satisfy the requirement. The single-cut efficiencies for vertexing-cuts DV fitting variables are listed. The variables $\overline{\Delta t}$ and $\sigma_{\Delta \mathrm{t}}$ are highly degenerate with $\bar{t}$ and $\sigma_{\mathrm{t}}$, and are not used here. ${ }^{8}$ After multiplying $N_{\text {ini }}$ by the cut efficiencies in the " 5 tracks" row, the " $\epsilon_{\mathrm{vtc}}$ " row and the " $\left(d_{0}>0.03 \mathrm{~m}\right)^{5}$ " row, we obtain the final event number $N_{\text {fin }}$.

For the QCD background, we apply a partial set of vertexing-cuts on $\Delta \mathrm{D}_{\min }, \sigma_{\mathrm{t}}$, and $\sigma_{\mathrm{z}}$. The cuts with $(*)$ are correlated with transverse impact parameter $d_{0}$ cut. Hence, they are not included in " $\epsilon_{\mathrm{vtc}}$ " to avoid double counting. ${ }^{9}$ Furthermore, we apply the single cut efficiency $\epsilon\left(1\right.$ track $\left.d_{0}>0.03 \mathrm{~m}\right)$ five times as an estimate of the efficiency requiring all the five tracks with $d_{0}>0.03 \mathrm{~m}$. We found the background the number of the events for $j j$ and $b \bar{b}$ are $5.7 \times 10^{-3}$ and $2.9 \times 10^{-4}$ respectively. We have demonstrated that $d_{0}$ cuts on different tracks are approximate independent, as discussed in detail in the previous section and the appendix. Nevertheless, one might still worry that cutting on five tracks is too aggressive. We also consider, as an alternative, cutting on only four tracks together with a stronger cut $d_{0}>0.05 \mathrm{~m}$. In this case, the single-cut efficiency for $d_{0}>0.05 \mathrm{~m}$ is about

\footnotetext{
${ }^{8}$ For a general discussion on effectiveness of time-delay variable for a broad class of LLP signatures, see ref. [103].

${ }^{9}$ One can apply them in an experimental search and it will help to further suppress the QCD background.
} 
$2.5 \times 10^{-5}$ for QCD backgrounds. After applying $\left(d_{0}>0.05 \mathrm{~m}\right)^{4}$, QCD background can be suppressed down to $\sim 10^{-5}$, which works equally well.

For the fake-track background, we multiply the individual efficiency for each variable in the vertexing-cuts and obtain $\epsilon_{\mathrm{vtc}}=4.0 \times 10^{-13}$. Requiring all five tracks with $d_{0}>0.03 \mathrm{~m}$ can suppress the background further by a factor of 0.34 , leaving only 0.14 events. We note that, even though we did not include it in this analysis, the fake-track has to match the track information with the HGCAL calorimeter energy deposit [96], which can further suppress the fake-track background.

In summary, both the QCD background and the fake-track background can be suppressed to be smaller than one event during the lifetime of the HL-LHC. The suppression for the QCD background mainly comes from requiring large track displacement, while displaced vertex reconstruction is mainly responsible for suppressing the fake-track background.

For the signal, the full set of vertexing-cuts are applied with a total efficiency of $\epsilon_{\mathrm{vtc}}=$ 0.34 and 0.24 for $m_{X}=20 \mathrm{GeV}$ and $50 \mathrm{GeV}$, respectively. Applying $d_{0}$ cuts on all the tracks reduces the signal further. Multiplying the sub-sequential overall cut efficiency from the columns with 5 tracks, $\epsilon_{\mathrm{vtc}}$ and $\left(d_{0}>0.03 \mathrm{~m}\right)^{5}$, one obtains the total cut efficiency for the signal. This is the exact signal efficiency for applying all the cuts. The remaining signal events as a function of branching ratio $\mathrm{BR}(h \rightarrow X X)$ is given in the last row. Heavier $X$ has higher efficiency for several reasons. First, heavier $X$ moves slower, leading to a larger probability of decaying before reaching HGCAL for a fixed proper lifetime. Second, lighter $X$ has only a slightly better efficiency under the $\epsilon_{\mathrm{vtc}}$ cut. Last, lighter $X$ has a lower $d_{0}$ cut efficiency, because the tracks tend to be collimated with the direction of $X$. Therefore, the search is more sensitive to heavier $X$. For the VBF channel, the distributions of vertexing-cuts variables in figure 11 and transverse impact parameter $d_{0}$ in figure 12 are similar to those of the ggF signal. Comparing with the ggF signal, the sensitivity in the VBF channel is weaker by about two orders of magnitude due to the smaller cross-section and the stringent VBF trigger threshold.

\subsection{The reach}

The results in the previous sections allow the determination of the potential in the search for new physics. In this section, we present the results for both the ggF and VBF channels. In figure 1, we show the projected sensitivity in the Higgs exotic decay into LLPs branching fractions, $B R(h \rightarrow X X)$, as a function of the proper lifetime of the LLP for both channels. The VBF search, shown in the left panel, represents a very conservative strategy with the existing VBF trigger. The ggF search, on the right panel, requires a dedicated displaced trigger. The solid line on the bottom of the color shaded region indicates the reach using a 5-displaced-track trigger, while for the solid line on the top of the shaded region, an additional $H_{T}>100 \mathrm{GeV}$ cut is employed. It represents a more conservative version of the displaced trigger, and consequently, it decreases the sensitivity by a factor of 10 . The best reach for $\mathrm{VBF}$ channel is about $\mathrm{BR}(h \rightarrow X X) \sim \mathcal{O}\left(10^{-4}\right)$, with the LLP lifetime of $c \tau_{X} \sim 0.1-1$ meters, while for the ggF channel it is about $\mathrm{BR}(h \rightarrow X X) \sim \mathcal{O}\left(10^{-5}-10^{-6}\right)$ for a similar lifetime. Alternatively, for an LLP with $c \tau_{X} \sim 10^{3}$ meters, the HGCAL based search should be able to probe $\operatorname{BR}(h \rightarrow X X)$ down to a few $\times 10^{-4}\left(10^{-2}\right)$ in the $g g F(\mathrm{VBF})$ channels, respectively. 
For comparison, we show the limits from existing searches for our benchmark signal model in figure 1. For very small $c \tau_{X}$, the best limits come from the ATLAS search for the prompt $h \rightarrow X X \rightarrow 4 b$, at $13 \mathrm{TeV}$ with $36.1 \mathrm{fb}^{-1}$ [80]. A short lifetime of $X$ is allowed by the b-tagging algorithm, with maximal sensitivity for $c \tau_{X} \sim 0.5 \mathrm{~mm}$. For $c \tau_{X}$ between $\left\{10^{-2}, 10^{3}\right\} \mathrm{m}$, there are several ATLAS searches using $13 \mathrm{TeV}$ data. One is based on the muon spectrometer (MS) with $36.1 \mathrm{fb}^{-1}$ [18]. The other uses the low- $E_{T}$ calorimeter energy ratio trigger, with $10.8 \mathrm{fb}^{-1}$ [14]. In the gap for LLP lifetime around $\mathrm{cm}$, the displaced jet searches can be sensitive. A recent CMS search based on displaced vertex in the tracker system with $139 \mathrm{fb}^{-1}$ obtained limits at the level of $10^{-1}-10^{-2}$ [13] for LLP decay $X \rightarrow \bar{b}$. Though its limit on $X \rightarrow \bar{d} d$ is about 10 times better due to one reconstructed secondary vertex requirement.

Since we are using HL-LHC with integrated luminosity $3 \mathrm{ab}^{-1}$, it is not a fair comparison for the existing limits. One can scale up the results of those search to $3 \mathrm{ab}^{-1}$. The sensitivity gain is proportional to the square root of the luminosity increase since those searches have non-zero backgrounds. As a result, the gain from luminosity ranges from 9.1 to 4.8. The other improvements we have compared to the existing searches are from both trigger and background suppression. The trigger efficiencies are 0.033, 0.040 and 0.21 for signals $m_{X}=50 \mathrm{GeV}$ for VBF channel, ggF channel with $H_{T}$ cut and ggF channel without $H_{T}$ cut respectively. They have included the requirement of track arrival at HGCAL. Therefore, it is easy to see that the novel trigger from [91] provides an improvement about a factor of 5 . The last improvement comes from the ability of driving the background to a negligible level. From figure 1, for long lifetime case (e.g. $c \tau=1 \mathrm{~km}$ ), the sensitivity of ggF channel with the novel trigger is better than the existing limit (e.g. "ATLAS-MS") by a factor of about 1000. The improvement from the high luminosity is about 9 , and the novel trigger contributes a factor of 5 . The improvement from the background suppression using vertexing and track information at HGCAL contributes a factor of about 20, which is one of the dominant factors of our enhanced sensitivity.

\section{Conclusion}

High granularity calorimeters offer new opportunities for the search of the long-lived particle. In this work, we study the potential reach for the long-lived particle signal based upon a new search mainly relying on the HGCAL upgrade of the CMS detector. We present results based on both the more conservative traditional VBF trigger and a pair of novel displaced track triggers. Based on a simplified modeling of the signal and background of this new approach, we carefully devised kinematical cuts and estimated the size of the leading backgrounds. HGCAL provides the shower direction and timing information with unprecedented precision, enabling us to view them as "tracks". We find that the QCD background is mostly prompt, which can be suppressed effectively by requiring a large transverse impact parameter for multiple tracks, after applying the vertex-cuts removing the SM metastable mesons. Another major source of background is the fake-track background, which comes from mis-connected hits in the detector. The resulting tracks have a random distribution, typically with a large transverse impact parameter and hence requires additional selection. We take advantage of the fact that these tracks to rarely fit 
in a common vertex and design a set of corresponding vertexing-cuts to suppress such backgrounds. Using these selections, combined with our different trigger considerations, we obtained our projections of the HL-LHC sensitivities for Higgs decaying to LLPs at HGCAL that improves the current reach by $2-4$ orders of magnitude.

Finally, we note here our study is rather conservative in many aspects. leaving potentially large room for further improvement.

- For the QCD background and the signal, the most relevant parameter of the HGCAL detector is its angular resolution. In this study, we use the standalone angular resolution from HGCAL. In practice, the track trajectory can be detected by both the tracking system and the tracker inside the HGCAL. Combining the two can further improve the angular resolution. This will result in a better DV fitting and enhance the suppression of the QCD background.

- We require the LLP to decay way before reaching the HGCAL detector, leaving at least five hits in the outer layer of the tracking system. With a detailed understanding of the showering behavior of the background, novel searches for LLP decaying within the HGCAL can also be sensitive. This will enable an HGCAL standalone trigger, and enlarge the decay volume for the LLP (hence the reach in $c \tau_{X}$ ) by a factor of a few.

- In our selection cuts, we have left a large room for improvement. For instance, we did not fully utilize the timing information of the displaced vertices. This is due to our lack of understanding of fake-track behavior in the timing dimension. A full-fledged $4 \mathrm{D}$ vertex fit could result in a much more powerful suppression of the background.

- For LLPs with lower lifetime, our cuts are not optimal. Three of our cuts are mainly responsible for reducing the signal efficiency at lower lifetimes: these are $d_{0}>0.05 \mathrm{~m}$, which is 10-50 times larger than the normal cuts on displaced tracks; $\bar{t}>1 \mathrm{~ns}$, which effectively requires the signal to decay after traveling $30 \mathrm{~cm} ;|\bar{z}|>0.4 \mathrm{~m}$, which again requires the signal to decay after traveling more than $40 \mathrm{~cm}$. Many of these cuts can be adjusted and make the search more effective.

\section{Acknowledgments}

We thank Jared Evans, Yuri Gershtein, Simon Knapen, Felix Kling for helpful discussion. JL acknowledges support by an Oehme Fellowship. ZL is supported in part by the NSF under Grant No. PHY1620074 and by the Maryland Center for Fundamental Physics. XPW is supported by the U.S. Department of Energy under Contract No. DE-AC02-06CH11357. LTW is supported by the DOE grant DE-SC0013642. ZL and LTW acknowledge the hospitality of the Kavli Institute for Theoretical Physics, UC Santa Barbara, during the "Origin of the Vacuum Energy and Electroweak Scales" workshop supported by the NSF grant PHY-174958. ZL and LTW would also like to thank Aspen Center for Physics (supported by NSF grant PHY-1607611) for support from their programs and providing the environment for collaboration. 
(a)

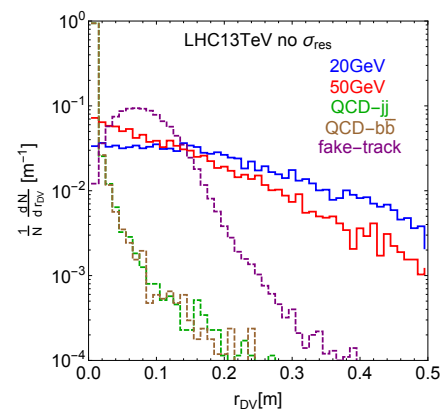

(b)

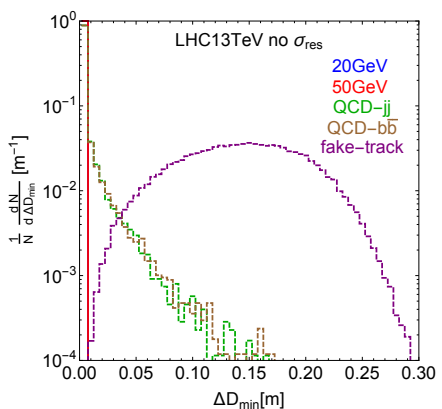

(c)

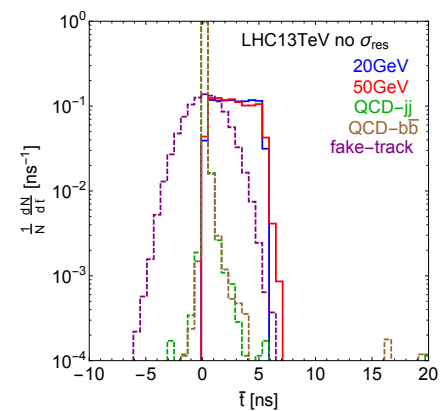

(f)

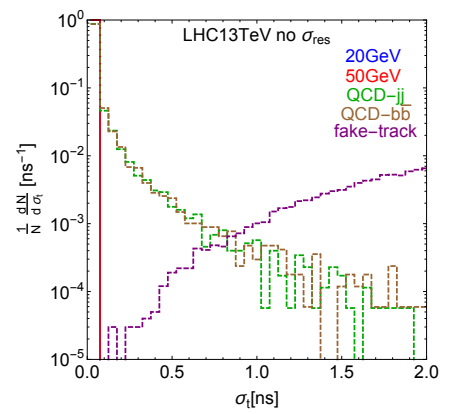

(d)

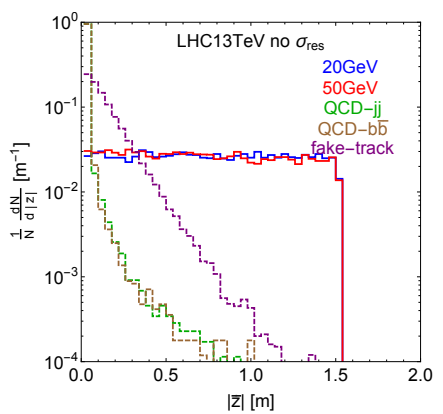

$(\mathrm{g})$

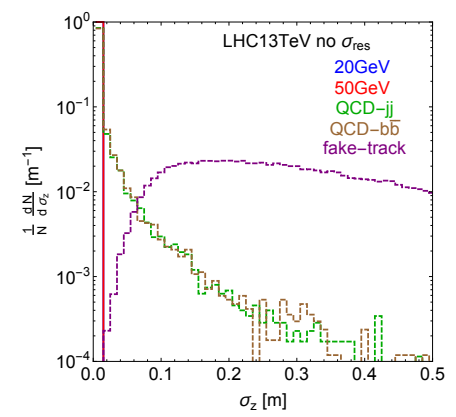

(e)

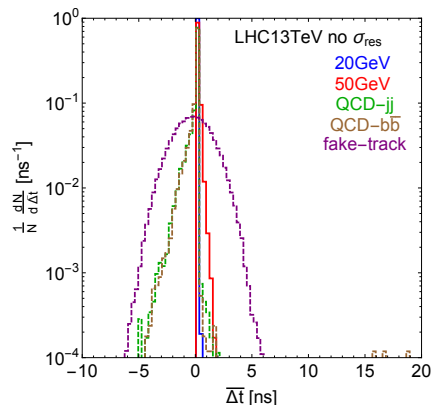

(h)

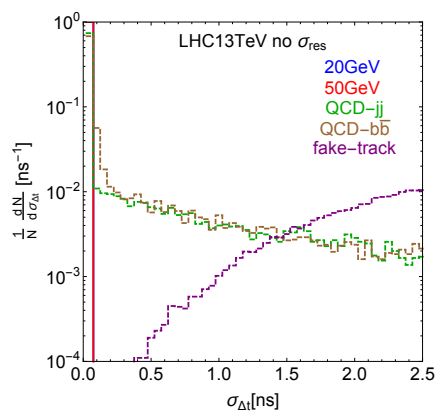

Figure 7. The kinetic variable distributions for the QCD background, fake-track background and the signal without the angular resolution effect included. The variables and definitions are the same as figure 5 .

\section{A Supportive figures and tables}

We put the supportive figures and tables in the appendix to avoid redundancy in the main text while keeping helpful information to the readers.

In figure 7, kinetic variable distributions for the QCD background, fake-track background and the signal are shown without the angular resolution effect included. This is a sanity check for figure 5 which has included the angular resolution effect. For the distribution of $\Delta \mathrm{D}_{\min }, \sigma_{\mathrm{t}}, \sigma_{\mathrm{z}}$ and $\sigma_{\Delta \mathrm{t}}$, the signals are exactly at 0 while the QCD background are peaked at 0 . It shows that the DV fitting algorithm has worked well and found the expected true vertex. 

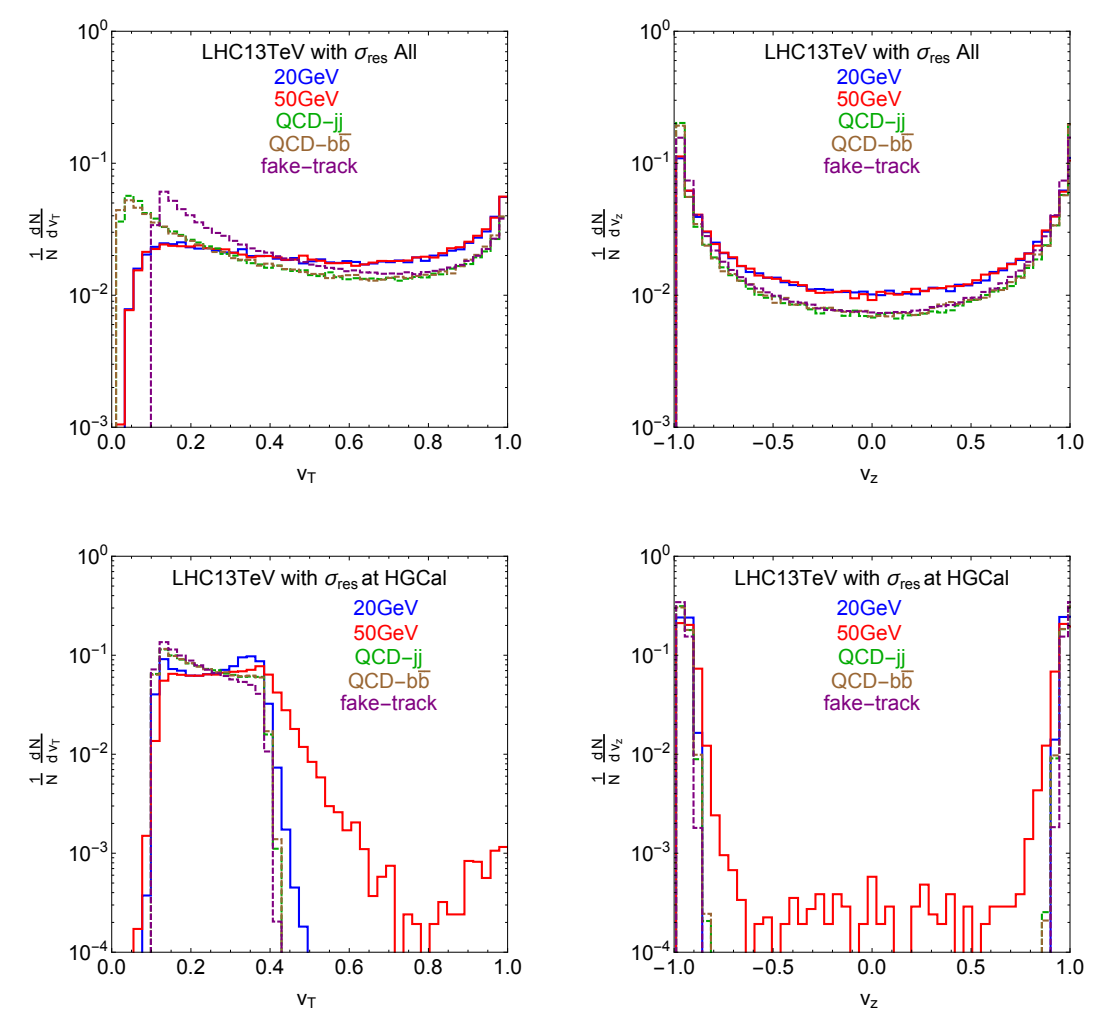

Figure 8. The distributions of transverse velocity $v_{T}$ and longitudinal velocity $v_{z}$ for the tracks without (upper panel) and with (lower panel) the requirement to arrive at HGCAL.

In figure 8, we plot the distribution of $v_{T}$ and $v_{z}$ for the tracks with and without the requirement to arrive at HGCAL. Moreover, we require the track should not hit the barrel electrocalorimeter. In the upper panel, it is clear to see that without requiring arriving at HGCAL, the $\left|v_{z}\right|$ distribution for all the signal and background have a peak around 1, while a flat valley in the middle. This reflects the distribution of the track zenith angle $\theta$. Once requiring arriving at HGCAL, we can see that the $v_{T}$ for signal and backgrounds are dominated by small values, e.g., $0.1 \sim 0.4$. The reason is that HGCAL is a forward detector, which picks the forward tracks. Therefore, the $v_{T}$ is forced to be small.

In figure 9 , we show the distribution of $\Delta \phi$ for the tracks in the DV fitting procedure. The QCD background and the signal have a similar distribution, peaked with $\Delta \phi=0$ because they both have a common vertex. $\Delta \phi$ comes from the angular resolution effect of HGCAL, which has a spread of about 0.02, which is a few times the angular resolution $\sigma_{\theta}$. For fake-track background, the distribution of $\Delta \phi$ has a reason smaller than order 1 . From the definition of $\Delta \phi$, its starting point (the reference point) is the closest point to the origin. Hence, the fitted DV should be enclosed within these reference points, as going far from the origin will lead to a bad fit. As a result, the movement in $\phi$ angle is not large from the starting point to DV.

In table 6 , we show the independence correlation table for multiple $d_{0}$ tracks for QCD dijet backgrounds without applying vertexing-cuts. This is an auxiliary check for table 2 . It has higher statistics and also shows the $d_{0}$ of different tracks are nicely independent under this condition. 


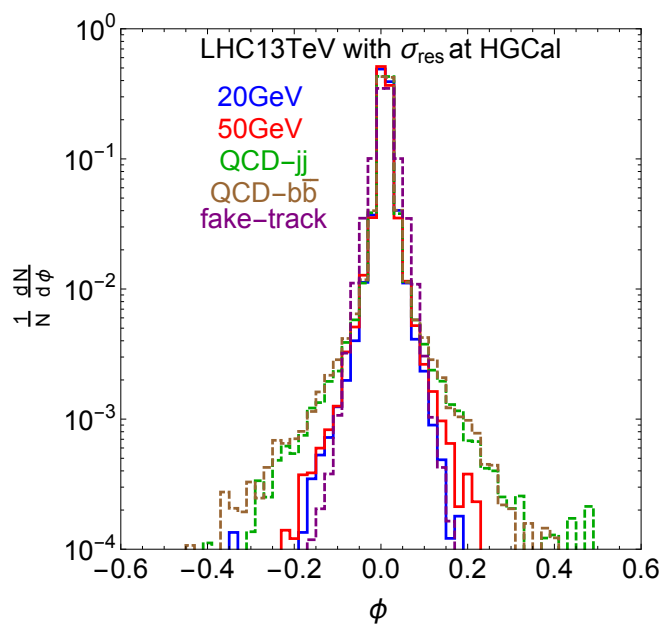

Figure 9. The distribution of $\Delta \phi$ for the tracks in the DV fitting procedure, where $\Delta \phi$ is the azimuthal angle change when moving from the reference point to the fitted DV.

\begin{tabular}{|c|c|c|c|c|c|}
\hline$j j$ dijets & $d_{0}>0.01 \mathrm{~m}$ & $d_{0}>0.015 \mathrm{~m}$ & $d_{0}>0.02 \mathrm{~m}$ & $d_{0}>0.025 \mathrm{~m}$ & $d_{0}>0.03 \mathrm{~m}$ \\
\hline$\rho_{d}^{1}$ & $1.0 \pm 0.06$ & $1.0 \pm 0.008$ & $1.0 \pm 0.01$ & $1.0 \pm 0.015$ & $1.0 \pm 0.02$ \\
\hline$\rho_{d}^{2}$ & $1.0 \pm 0.01$ & $0.98 \pm 0.016$ & $0.96 \pm 0.025$ & $0.88 \pm 0.038$ & $0.74 \pm 0.053$ \\
\hline$\rho_{d}^{3}$ & $0.99 \pm 0.018$ & $0.98 \pm 0.032$ & $0.90 \pm 0.062$ & $0.90 \pm 0.15$ & $1.3 \pm 0.65$ \\
\hline$\rho_{d}^{4}$ & $0.97 \pm 0.027$ & $1.0 \pm 0.07$ & $0.75 \pm 0.14$ & - & - \\
\hline$\rho_{d}^{5}$ & $0.95 \pm 0.04$ & $0.95 \pm 0.14$ & - & - & - \\
\hline$b \bar{b}$ dijets & $d_{0}>0.01 \mathrm{~m}$ & $d_{0}>0.015 \mathrm{~m}$ & $d_{0}>0.02 \mathrm{~m}$ & $d_{0}>0.025 \mathrm{~m}$ & $d_{0}>0.03 \mathrm{~m}$ \\
\hline$\rho_{d}^{1}$ & $1.0 \pm 0.06$ & $1.0 \pm 0.008$ & $1.0 \pm 0.01$ & $1.0 \pm 0.015$ & $1.0 \pm 0.02$ \\
\hline$\rho_{d}^{2}$ & $1.0 \pm 0.01$ & $1.0 \pm 0.017$ & $0.97 \pm 0.026$ & $0.89 \pm 0.40$ & $0.76 \pm 0.056$ \\
\hline$\rho_{d}^{3}$ & $0.98 \pm 0.018$ & $0.95 \pm 0.032$ & $0.93 \pm 0.066$ & $0.69 \pm 0.1$ & - \\
\hline$\rho_{d}^{4}$ & $0.97 \pm 0.027$ & $0.93 \pm 0.06$ & $1.1 \pm 0.24$ & - & - \\
\hline$\rho_{d}^{5}$ & $0.94 \pm 0.04$ & $0.81 \pm 0.11$ & - & - & - \\
\hline
\end{tabular}

Table 6. The correlation table for multiple $d_{0}$ tracks for QCD dijet backgrounds without applying the vertexing-cuts. The symbol "- " means no events left and the number in "( )" indicates the small number of statistics after the cuts. When increasing to multiple tracks and larger $d_{0}$ cuts, there are less events thus the result suffers from larger statistical fluctuations. It is an auxiliary check for table 2 that is after applying the vertexing-cuts.

The table 7 shows the independence correlation table for vertexing-cuts variables for fake-track backgrounds, but with a weaker set of cuts comparing with table 3 . We can see that most of the correlations are around 1 (approximate independent), with some results are 4.8 and 20 which are conservative. With the table 3 and table 7 , it indicates that the estimate of fake-track background by multiplying each of these efficiency should be considered as conservative. 


\begin{tabular}{|c|c|c|c|c|c|c|}
\hline fake-track & $\mathrm{r}_{\mathrm{DV}}>0.05 \mathrm{~m}$ & $\Delta \mathrm{D}_{\min }<0.05 \mathrm{~m}$ & $\bar{t}>2 \mathrm{~ns}$ & $\sigma_{\mathrm{t}}<0.5 \mathrm{~ns}$ & $|\bar{z}|>0.4 \mathrm{~m}$ & $\sigma_{\mathrm{z}}<0.1 \mathrm{~m}$ \\
\hline $\mathrm{r}_{\mathrm{DV}}>0.05 \mathrm{~m}$ & & $0.814 \pm 0.006$ & $0.95 \pm 0.004$ & $1.03 \pm 0.08$ & $0.751 \pm 0.004$ & $5.58 \pm 0.07$ \\
\hline$\Delta \mathrm{D}_{\min }<0.05 \mathrm{~m}$ & $0.814 \pm 0.006$ & & $0.95 \pm 0.02$ & $1.19 \pm 0.36$ & $0.65 \pm 0.01$ & $2.37 \pm 0.09$ \\
\hline $\bar{t}>2 \mathrm{~ns}$ & $0.95 \pm 0.004$ & $0.95 \pm 0.02$ & & $0.40 \pm 0.04$ & $0.533 \pm 0.005$ & $1.19 \pm 0.02$ \\
\hline$\sigma_{\mathrm{t}}<0.5 \mathrm{~ns}$ & $1.03 \pm 0.08$ & $1.19 \pm 0.36$ & $0.40 \pm 0.04$ & & $2.49 \pm 0.80$ & $0.77 \pm 0.16$ \\
\hline$|\bar{z}|>0.4 \mathrm{~m}$ & $0.751 \pm 0.004$ & $0.65 \pm 0.01$ & $0.533 \pm 0.005$ & $2.49 \pm 0.80$ & & $16.23 \pm 0.94$ \\
\hline$\sigma_{\mathrm{z}}<0.1 \mathrm{~m}$ & $5.58 \pm 0.07$ & $2.37 \pm 0.09$ & $1.19 \pm 0.02$ & $0.77 \pm 0.16$ & $16.23 \pm 0.94$ & \\
\hline
\end{tabular}

Table 7. The correlation table for vertexing-cuts variables for fake-track backgrounds. These cuts are weaker than the cuts in table 3 .
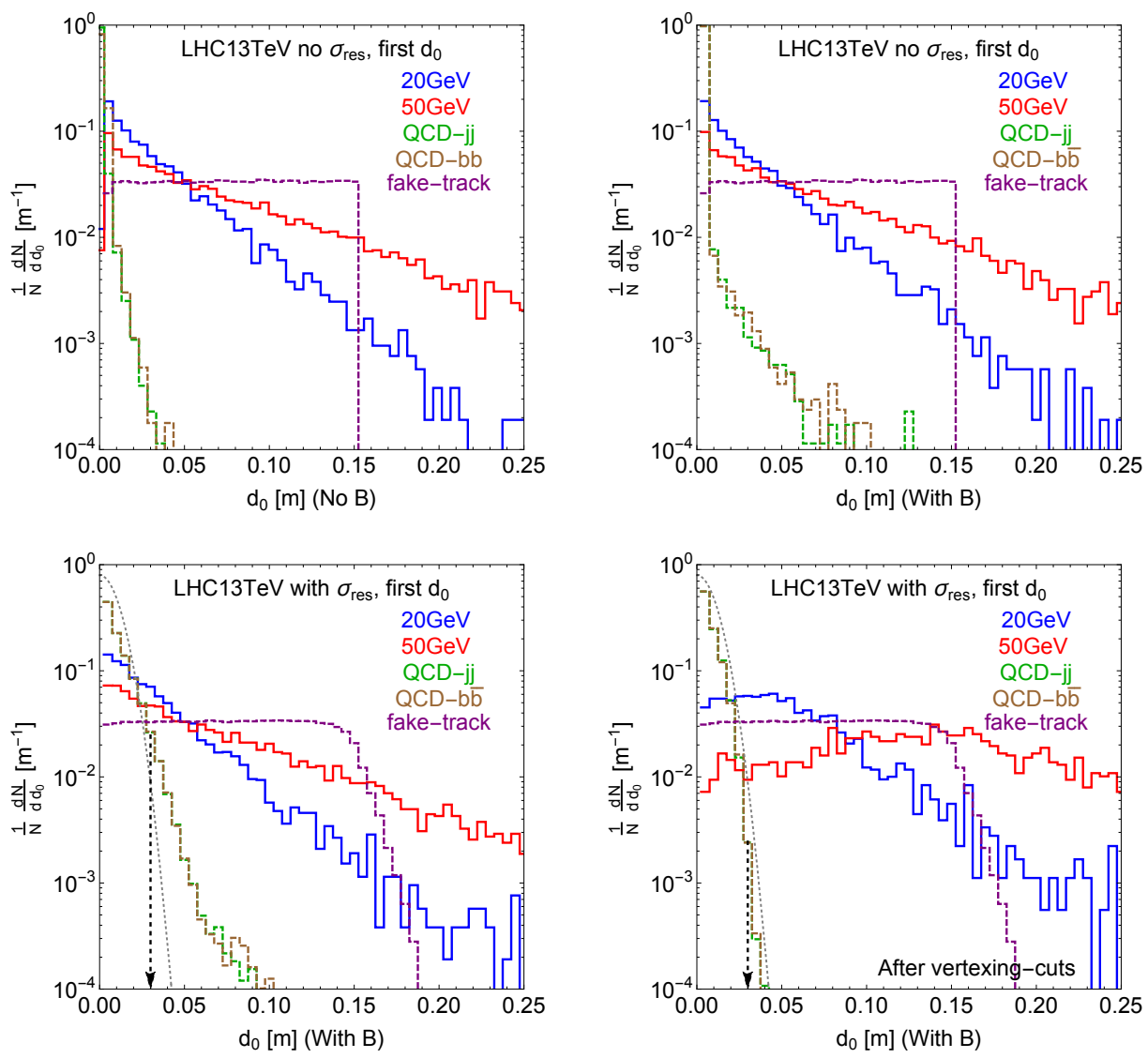

Figure 10. The transverse impact parameter $d_{0}$ distributions for QCD background, fake-track background and the signal. This figure is similar to figure 6 , but only the leading track distribution is displayed.

In figure 10, we show the transverse impact parameter $d_{0}$ distribution of the leading track for QCD background, fake-track background, and the signal. This figure is similar to figure 6 , but with only the leading track included.

In figure 11 and figure 12 , the kinetic variables and $d_{0}$ distributions for VBF channel are given. One can see that the distributions of the VBF channel are similar to the ggF channel. 

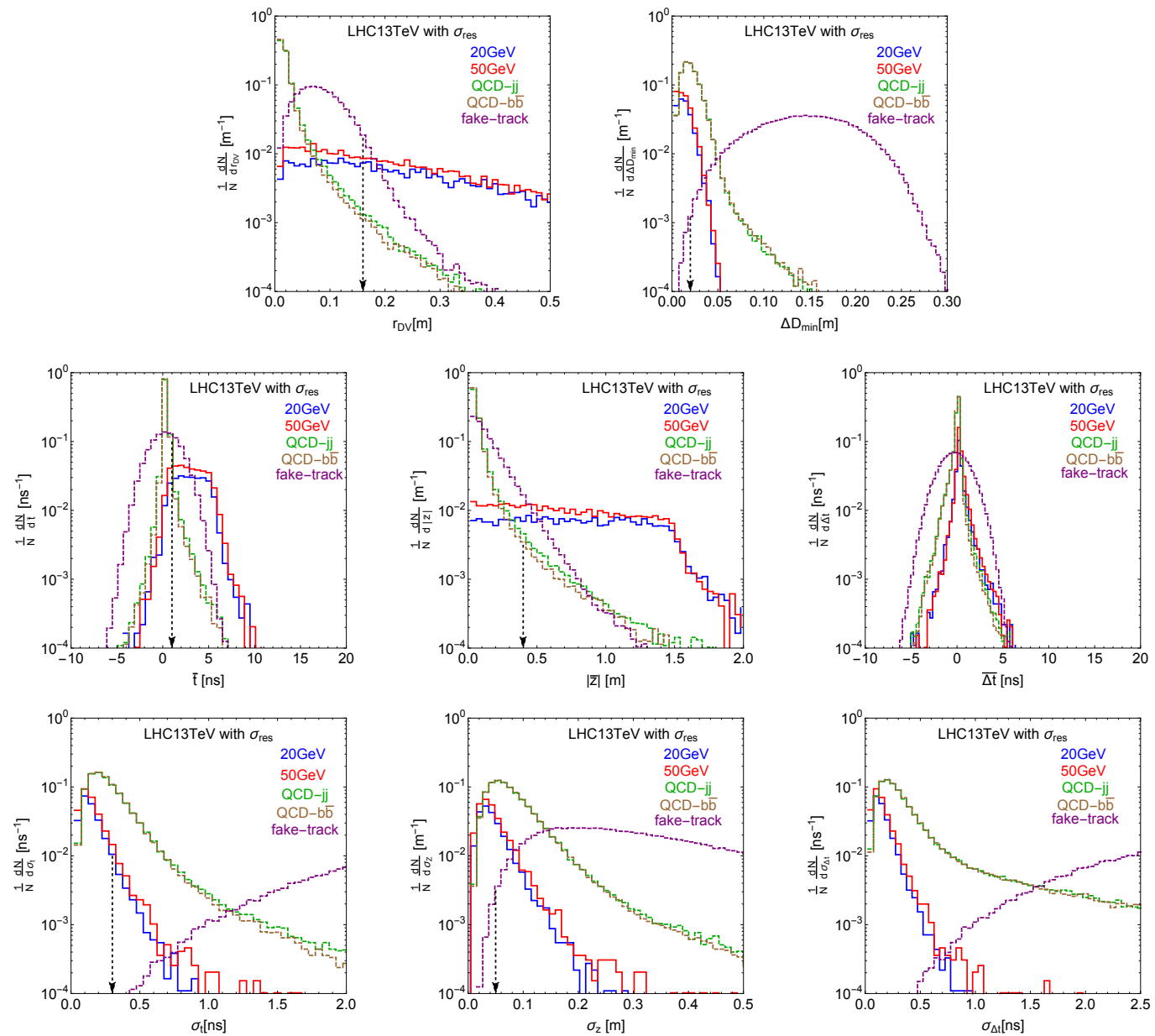

Figure 11. (VBF channel) The kinetic variable distributions for the QCD background, fake-track background and the signal with angular resolution effect included. The parameter setup is the same as the $\mathrm{ggF}$ channel in figure 5 .
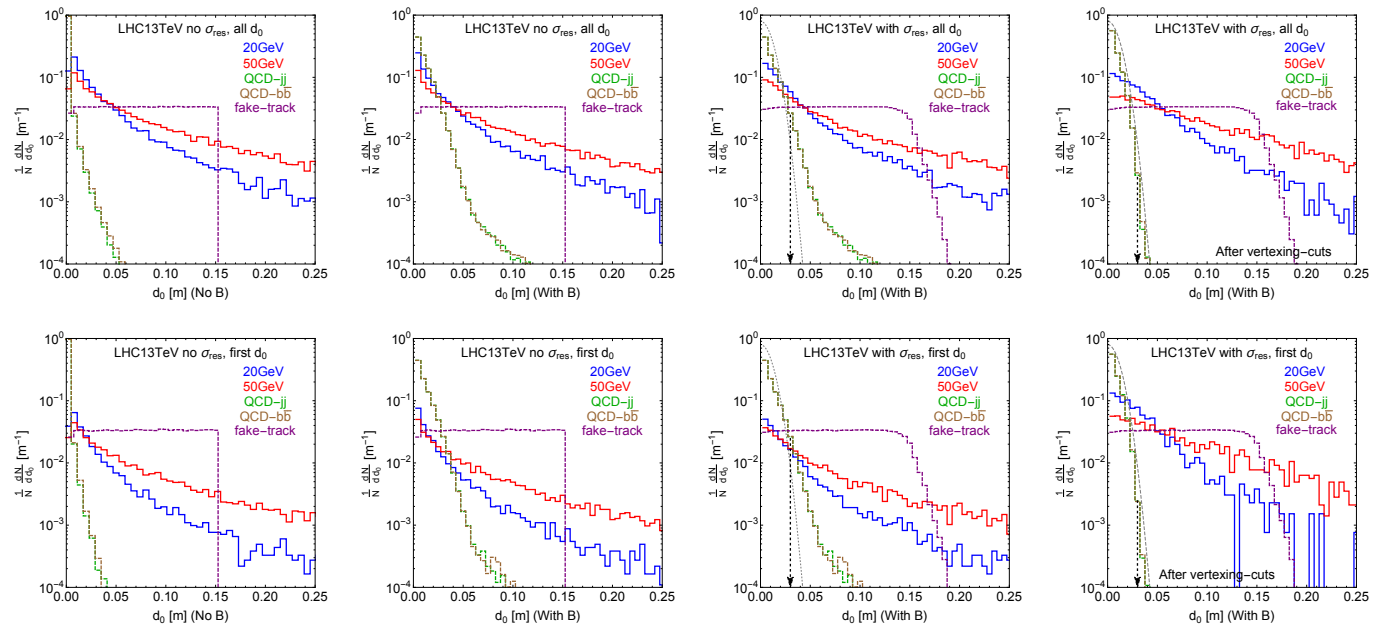

Figure 12. (VBF channel) The distributions for transverse impact parameter $d_{0}$ for QCD background, fake-track background and the signal. The parameter setup is the same as the ggF channel in figure 6 and figure 10 . 
Open Access. This article is distributed under the terms of the Creative Commons Attribution License (CC-BY 4.0), which permits any use, distribution and reproduction in any medium, provided the original author(s) and source are credited.

\section{References}

[1] J. Alimena et al., Searching for long-lived particles beyond the standard model at the Large Hadron Collider, J. Phys. G 47 (2020) 090501 [arXiv: 1903. 04497] [InSPIRE].

[2] ATLAS collaboration, Search for nonpointing and delayed photons in the diphoton and missing transverse momentum final state in $8 \mathrm{TeV}$ pp collisions at the LHC using the ATLAS detector, Phys. Rev. D 90 (2014) 112005 [arXiv:1409.5542] [INSPIRE].

[3] CMS collaboration, Search for long-lived particles using delayed photons with proton-proton collisions at $\sqrt{s}=13 \mathrm{TeV}$, CMS-PAS-EXO-19-005 (2019).

[4] ATLAS collaboration, Search for charginos nearly mass degenerate with the lightest neutralino based on a disappearing-track signature in pp collisions at $\sqrt{s}=8 \mathrm{TeV}$ with the ATLAS detector, Phys. Rev. D 88 (2013) 112006 [arXiv:1310.3675] [INSPIRE].

[5] ATLAS collaboration, Search for long-lived charginos based on a disappearing-track signature in pp collisions at $\sqrt{s}=13$ TeV with the ATLAS detector, JHEP 06 (2018) 022 [arXiv: 1712.02118] [INSPIRE].

[6] CMS collaboration, Search for disappearing tracks as a signature of new long-lived particles in proton-proton collisions at $\sqrt{s}=13 \mathrm{TeV}$, JHEP 08 (2018) 016 [arXiv:1804.07321] [INSPIRE].

[7] ATLAS collaboration, Search for massive, long-lived particles using multitrack displaced vertices or displaced lepton pairs in pp collisions at $\sqrt{s}=8 \mathrm{TeV}$ with the ATLAS detector, Phys. Rev. D 92 (2015) 072004 [arXiv:1504.05162] [INSPIRE].

[8] ATLAS collaboration, Search for long-lived, massive particles in events with displaced vertices and missing transverse momentum in $\sqrt{s}=13 \mathrm{TeV}$ pp collisions with the ATLAS detector, Phys. Rev. D 97 (2018) 052012 [arXiv:1710.04901] [INSPIRE].

[9] CMS collaboration, Search for long-lived particles that decay into final states containing two electrons or two muons in proton-proton collisions at $\sqrt{s}=8$ TeV, Phys. Rev. D 91 (2015) 052012 [arXiv:1411.6977] [INSPIRE].

[10] CMS collaboration, Search for long-lived particles with displaced vertices in multijet events in proton-proton collisions at $\sqrt{s}=13 \mathrm{TeV}$, Phys. Rev. D 98 (2018) 092011 [arXiv:1808.03078] [INSPIRE].

[11] CMS collaboration, Search for long-lived particles decaying into displaced jets in proton-proton collisions at $\sqrt{s}=13 \mathrm{TeV}$, Phys. Rev. D 99 (2019) 032011 [arXiv: 1811.07991] [INSPIRE].

[12] CMS collaboration, Search for displaced leptons in the e- $\mu$ channel, CMS-PAS-EXO-16-022 (2016).

[13] CMS collaboration, Search for long-lived particles decaying into displaced jets, CMS-PAS-EXO-19-021 (2020).

[14] ATLAS collaboration, Search for long-lived neutral particles in pp collisions at $\sqrt{s}=13$ $\mathrm{TeV}$ that decay into displaced hadronic jets in the ATLAS calorimeter, Eur. Phys. J. C 79 (2019) 481 [arXiv: 1902.03094] [INSPIRE]. 
[15] CMS collaboration, Search for decays of stopped exotic long-lived particles produced in proton-proton collisions at $\sqrt{s}=13 \mathrm{TeV}$, JHEP 05 (2018) 127 [arXiv:1801.00359] [INSPIRE].

[16] ATLAS collaboration, Search for long-lived, weakly interacting particles that decay to displaced hadronic jets in proton-proton collisions at $\sqrt{s}=8$ TeV with the ATLAS detector, Phys. Rev. D 92 (2015) 012010 [arXiv:1504.03634] [INSPIRE].

[17] ATLAS collaboration, Search for long-lived particles in final states with displaced dimuon vertices in pp collisions at $\sqrt{s}=13$ TeV with the ATLAS detector, Phys. Rev. D 99 (2019) 012001 [arXiv: 1808.03057] [INSPIRE].

[18] ATLAS collaboration, Search for long-lived particles produced in pp collisions at $\sqrt{s}=13$ TeV that decay into displaced hadronic jets in the ATLAS muon spectrometer, Phys. Rev. D 99 (2019) 052005 [arXiv: 1811.07370] [INSPIRE].

[19] J. Heisig and J. Kersten, Long-lived staus from strong production in a simplified model approach, Phys. Rev. D 86 (2012) 055020 [arXiv: 1203.1581] [InSPIRE].

[20] K. Barry, P.W. Graham and S. Rajendran, Displaced vertices from R-parity violation and baryogenesis, Phys. Rev. D 89 (2014) 054003 [arXiv:1310.3853] [InSPIRE].

[21] J.C. Helo, M. Hirsch and S. Kovalenko, Heavy neutrino searches at the LHC with displaced vertices, Phys. Rev. D 89 (2014) 073005 [Erratum ibid. 93 (2016) 099902] [arXiv: 1312.2900] [INSPIRE].

[22] Y. Cui and B. Shuve, Probing baryogenesis with displaced vertices at the LHC, JHEP 02 (2015) 049 [arXiv: 1409.6729] [InSPIRE].

[23] SHIP collaboration, A facility to Search for Hidden Particles (SHiP) at the CERN SPS, arXiv: 1504.04956 [INSPIRE].

[24] S. Alekhin et al., A facility to Search for Hidden Particles at the CERN SPS: the SHiP physics case, Rept. Prog. Phys. 79 (2016) 124201 [arXiv:1504.04855] [INSPIRE].

[25] R.T. Co, F. D'Eramo, L.J. Hall and D. Pappadopulo, Freeze-in dark matter with displaced signatures at colliders, JCAP 12 (2015) 024 [arXiv: 1506.07532] [INSPIRE].

[26] Z. Liu and B. Tweedie, The fate of long-lived superparticles with hadronic decays after LHC Run 1, JHEP 06 (2015) 042 [arXiv: 1503.05923] [INSPIRE].

[27] J.A. Evans and J. Shelton, Long-lived staus and displaced leptons at the LHC, JHEP 04 (2016) 056 [arXiv: 1601.01326] [INSPIRE].

[28] E. Accomando, L. Delle Rose, S. Moretti, E. Olaiya and C.H. Shepherd-Themistocleous, Novel SM-like Higgs decay into displaced heavy neutrino pairs in $\mathrm{U}(1)^{\prime}$ models, JHEP 04 (2017) 081 [arXiv: 1612.05977] [INSPIRE].

[29] P.S. Bhupal Dev, R.N. Mohapatra and Y. Zhang, Displaced photon signal from a possible light scalar in minimal left-right seesaw model, Phys. Rev. D 95 (2017) 115001 [arXiv: 1612.09587] [INSPIRE].

[30] LHCb collaboration, Search for Higgs-like bosons decaying into long-lived exotic particles, Eur. Phys. J. C 76 (2016) 664 [arXiv: 1609.03124] [InSPIRE].

[31] A. Coccaro, D. Curtin, H.J. Lubatti, H. Russell and J. Shelton, Data-driven model-independent searches for long-lived particles at the LHC, Phys. Rev. D 94 (2016) 113003 [arXiv: 1605.02742] [INSPIRE].

[32] S. Antusch, E. Cazzato and O. Fischer, Displaced vertex searches for sterile neutrinos at future lepton colliders, JHEP 12 (2016) 007 [arXiv: 1604.02420] [INSPIRE]. 
[33] O. Buchmueller et al., Simplified models for displaced dark matter signatures, JHEP 09 (2017) 076 [arXiv: 1704.06515] [INSPIRE].

[34] R. Mahbubani, P. Schwaller and J. Zurita, Closing the window for compressed Dark Sectors with disappearing charged tracks, JHEP 06 (2017) 119 [Erratum ibid. 10 (2017) 061] [arXiv: 1703. 05327] [INSPIRE].

[35] V.V. Khoze, A.D. Plascencia and K. Sakurai, Simplified models of dark matter with a long-lived co-annihilation partner, JHEP 06 (2017) 041 [arXiv:1702.00750] [INSPIRE].

[36] A. Ghosh, T. Mondal and B. Mukhopadhyaya, Heavy stable charged tracks as signatures of non-thermal dark matter at the LHC : a study in some non-supersymmetric scenarios, JHEP 12 (2017) 136 [arXiv:1706.06815] [INSPIRE].

[37] P.S.B. Dev, R.N. Mohapatra and Y. Zhang, Long Lived Light Scalars as Probe of Low Scale Seesaw Models, Nucl. Phys. B 923 (2017) 179 [arXiv:1703.02471] [inSPIRE].

[38] V.V. Gligorov, S. Knapen, M. Papucci and D.J. Robinson, Searching for long-lived particles: a compact detector for exotics at LHCb, Phys. Rev. D 97 (2018) 015023 [arXiv:1708.09395] [INSPIRE].

[39] J.L. Feng, I. Galon, F. Kling and S. Trojanowski, ForwArd Search ExpeRiment at the LHC, Phys. Rev. D 97 (2018) 035001 [arXiv:1708.09389] [InSPIRE].

[40] A. Abada, N. Bernal, M. Losada and X. Marcano, Inclusive displaced vertex searches for heavy neutral leptons at the LHC, JHEP 01 (2019) 093 [arXiv:1807.10024] [INSPIRE].

[41] J.A. Evans and M.A. Luty, Stopping quirks at the LHC, JHEP 06 (2019) 090 [arXiv: 1811.08903] [INSPIRE].

[42] G.D. Kribs, A. Martin, B. Ostdiek and T. Tong, Dark mesons at the LHC, JHEP 07 (2019) 133 [arXiv: 1809.10184] [INSPIRE].

[43] A. Berlin and F. Kling, Inelastic dark matter at the LHC lifetime frontier: ATLAS, CMS, LHCb, CODEX-b, FASER, and MATHUSLA, Phys. Rev. D 99 (2019) 015021 [arXiv: 1810.01879 ] [INSPIRE].

[44] I. Lara, D.E. López-Fogliani, C. Muñoz, N. Nagata, H. Otono and R. Ruiz De Austri, Looking for the left sneutrino LSP with displaced-vertex searches, Phys. Rev. D 98 (2018) 075004 [arXiv: 1804.00067] [INSPIRE].

[45] M. Nemevšek, F. Nesti and G. Popara, Keung-Senjanović process at the LHC: from lepton number violation to displaced vertices to invisible decays, Phys. Rev. D 97 (2018) 115018 [arXiv: 1801.05813] [inSPIRE].

[46] J.C. Helo, M. Hirsch and Z.S. Wang, Heavy neutral fermions at the high-luminosity LHC, JHEP 07 (2018) 056 [arXiv:1803.02212] [INSPIRE].

[47] G. Cottin, J.C. Helo and M. Hirsch, Searches for light sterile neutrinos with multitrack displaced vertices, Phys. Rev. D 97 (2018) 055025 [arXiv: 1801.02734] [InSPIRE].

[48] C. Kilic, S. Najjari and C.B. Verhaaren, Discovering the twin Higgs boson with displaced decays, Phys. Rev. D 99 (2019) 075029 [arXiv:1812.08173] [INSPIRE].

[49] P.S. Bhupal Dev and Y. Zhang, Displaced vertex signatures of doubly charged scalars in the type-II seesaw and its left-right extensions, JHEP 10 (2018) 199 [arXiv:1808.00943] [INSPIRE].

[50] L. Calibbi, L. Lopez-Honorez, S. Lowette and A. Mariotti, Singlet-doublet dark matter freeze-in: LHC displaced signatures versus cosmology, JHEP 09 (2018) 037 [arXiv: 1805.04423] [INSPIRE]. 
[51] FASER collaboration, FASER's physics reach for long-lived particles, Phys. Rev. D 99 (2019) 095011 [arXiv: 1811.12522] [inSPIRE].

[52] D. Curtin, K.R. Dienes and B. Thomas, Dynamical dark matter, MATHUSLA, and the lifetime frontier, Phys. Rev. D 98 (2018) 115005 [arXiv: 1809.11021] [INSPIRE].

[53] D. Curtin et al., Long-lived particles at the energy frontier: the MATHUSLA physics case, Rept. Prog. Phys. 82 (2019) 116201 [arXiv: 1806.07396] [InSPIRE].

[54] V.V. Gligorov, S. Knapen, B. Nachman, M. Papucci and D.J. Robinson, Leveraging the ALICE/L3 cavern for long-lived particle searches, Phys. Rev. D 99 (2019) 015023 [arXiv: 1810.03636] [INSPIRE].

[55] ATLAS collaboration, Search for long-lived, massive particles in events with displaced vertices and missing transverse momentum in $\sqrt{s}=13 \mathrm{TeV} p p$ collisions with the ATLAS detector, Phys. Rev. D 97 (2018) 052012 [arXiv:1710.04901] [INSPIRE].

[56] A. Das, N. Okada, S. Okada and D. Raut, Probing the seesaw mechanism at the $250 \mathrm{GeV}$ ILC, Phys. Lett. B 797 (2019) 134849 [arXiv:1812.11931] [InSPIRE].

[57] M. Drewes and J. Hajer, Heavy neutrinos in displaced vertex searches at the LHC and HL-LHC, JHEP 02 (2020) 070 [arXiv: 1903.06100] [INSPIRE].

[58] M. Du, Z. Liu and V.Q. Tran, Enhanced long-lived dark photon signals at the LHC, JHEP 05 (2020) 055 [arXiv: 1912.00422] [INSPIRE].

[59] J. Liu, Z. Liu, L.-T. Wang and X.-P. Wang, Seeking for sterile neutrinos with displaced leptons at the LHC, JHEP 07 (2019) 159 [arXiv: 1904.01020] [INSPIRE].

[60] MATHUSLA collaboration, Explore the lifetime frontier with MATHUSLA, 2020 JINST 15 C06026 [arXiv: 1901.04040] [INSPIRE].

[61] M. Drewes, A. Giammanco, J. Hajer and M. Lucente, New long-lived particle searches in heavy-ion collisions at the LHC, Phys. Rev. D 101 (2020) 055002 [arXiv:1905.09828] [INSPIRE].

[62] G. Aielli et al., Expression of interest for the CODEX-b detector, arXiv:1911.00481 [INSPIRE].

[63] M. Bauer, O. Brandt, L. Lee and C. Ohm, ANUBIS: proposal to search for long-lived neutral particles in CERN service shafts, arXiv:1909.13022 [INSPIRE].

[64] K. Bondarenko, A. Boyarsky, M. Ovchynnikov, O. Ruchayskiy and L. Shchutska, Probing new physics with displaced vertices: muon tracker at CMS, Phys. Rev. D 100 (2019) 075015 [arXiv: 1903.11918] [INSPIRE].

[65] A. Filimonova, R. Schäfer and S. Westhoff, Probing dark sectors with long-lived particles at BELLE II, Phys. Rev. D 101 (2020) 095006 [arXiv:1911.03490] [InSPIRE].

[66] J. Serra, S. Stelzl, R. Torre and A. Weiler, Hypercharged Naturalness, JHEP 10 (2019) 060 [arXiv: 1905. 02203] [INSPIRE].

[67] C. Argüelles, P. Coloma, P. Hernández and V. Muñoz, Searches for atmospheric long-lived particles, JHEP 02 (2020) 190 [arXiv:1910.12839] [INSPIRE].

[68] K. Cheung and Z.S. Wang, Probing long-lived particles at Higgs factories, Phys. Rev. D 101 (2020) 035003 [arXiv: 1911.08721] [INSPIRE].

[69] B. Bhattacherjee, S. Mukherjee and R. Sengupta, Study of energy deposition patterns in hadron calorimeter for prompt and displaced jets using convolutional neural network, JHEP 11 (2019) 156 [arXiv: 1904.04811] [INSPIRE]. 
[70] C.-W. Chiang, G. Cottin, A. Das and S. Mandal, Displaced heavy neutrinos from $Z^{\prime}$ decays at the LHC, JHEP 12 (2019) 070 [arXiv:1908.09838] [INSPIRE].

[71] A. Das, P.S.B. Dev and N. Okada, Long-lived TeV-scale right-handed neutrino production at the LHC in gauged $U(1)_{X}$ model, Phys. Lett. B 799 (2019) 135052 [arXiv:1906.04132] [INSPIRE].

[72] J. Li, T. Li, J. Pei and W. Zhang, The quirk trajectory, Eur. Phys. J. C 80 (2020) 651 [arXiv:2002.07503] [INSPIRE].

[73] C. Yuan, H. Zhang and Y. Zhao, Producing and detecting long-lived particles at different experiments at the LHC, arXiv:2004.08820 [INSPIRE].

[74] B.S. Acharya et al., Prospects of searches for long-lived charged particles with MoEDAL, Eur. Phys. J. C 80 (2020) 572 [arXiv:2004.11305] [inSPIRE].

[75] B. Bhattacherjee, S. Mukherjee, R. Sengupta and P. Solanki, Triggering long-lived particles in HL-LHC and the challenges in the rst stage of the trigger system, JHEP 08 (2020) 141 [arXiv: 2003. 03943] [INSPIRE].

[76] B. Shuve and D. Tucker-Smith, Baryogenesis and dark matter from freeze-in, Phys. Rev. D 101 (2020) 115023 [arXiv: 2004.00636] [INSPIRE].

[77] J. Alimena, Y. Iiyama and J. Kieseler, Fast convolutional neural networks for identifying long-lived particles in a high-granularity calorimeter, arXiv:2004.10744 [INSPIRE].

[78] D. Felea et al., Prospects for discovering supersymmetric long-lived particles with MoEDAL, Eur. Phys. J. C 80 (2020) 431 [arXiv:2001.05980] [inSPIRE].

[79] CMS collaboration, The phase-2 upgrade of the CMS endcap calorimeter, CERN-LHCC-2017-023 (2017).

[80] ATLAS collaboration, Search for the Higgs boson produced in association with a vector boson and decaying into two spin-zero particles in the $H \rightarrow a a \rightarrow 4 b$ channel in pp collisions at $\sqrt{s}=13 \mathrm{TeV}$ with the ATLAS detector, JHEP 10 (2018) 031 [arXiv:1806.07355] [INSPIRE].

[81] D. Curtin et al., Exotic decays of the 125 GeV Higgs boson, Phys. Rev. D 90 (2014) 075004 [arXiv: 1312.4992] [inSPIRE].

[82] M.J. Strassler and K.M. Zurek, Echoes of a hidden valley at hadron colliders, Phys. Lett. B 651 (2007) 374 [hep-ph/0604261] [INSPIRE].

[83] M.J. Strassler and K.M. Zurek, Discovering the Higgs through highly-displaced vertices, Phys. Lett. B 661 (2008) 263 [hep-ph/0605193] [INSPIRE].

[84] T. Han, Z. Si, K.M. Zurek and M.J. Strassler, Phenomenology of hidden valleys at hadron colliders, JHEP 07 (2008) 008 [arXiv:0712.2041] [INSPIRE].

[85] Z. Chacko, H.-S. Goh and R. Harnik, The twin Higgs: natural electroweak breaking from mirror symmetry, Phys. Rev. Lett. 96 (2006) 231802 [hep-ph/0506256] [INSPIRE].

[86] Z. Chacko, H.-S. Goh and R. Harnik, A twin Higgs model from left-right symmetry, JHEP 01 (2006) 108 [hep-ph/0512088] [InSPIRE].

[87] G. Burdman, Z. Chacko, H.-S. Goh and R. Harnik, Folded supersymmetry and the LEP paradox, JHEP 02 (2007) 009 [hep-ph/0609152] [INSPIRE].

[88] N. Craig, A. Katz, M. Strassler and R. Sundrum, Naturalness in the dark at the LHC, JHEP 07 (2015) 105 [arXiv: 1501.05310] [INSPIRE].

[89] C. Csáki, E. Kuflik, S. Lombardo and O. Slone, Searching for displaced Higgs boson decays, Phys. Rev. D 92 (2015) 073008 [arXiv:1508.01522] [InSPIRE]. 
[90] D. Curtin and C.B. Verhaaren, Discovering uncolored naturalness in exotic Higgs decays, JHEP 12 (2015) 072 [arXiv: 1506.06141] [INSPIRE].

[91] Y. Gershtein, CMS hardware track trigger: new opportunities for long-lived particle searches at the HL-LHC, Phys. Rev. D 96 (2017) 035027 [arXiv:1705.04321] [INSPIRE].

[92] J. Alwall et al., The automated computation of tree-level and next-to-leading order differential cross sections, and their matching to parton shower simulations, JHEP 07 (2014) 079 [arXiv: 1405.0301] [INSPIRE].

[93] T. Sjöstrand, S. Mrenna and P.Z. Skands, PYTHIA 6.4 physics and manual, JHEP 05 (2006) 026 [hep-ph/0603175] [INSPIRE].

[94] T. Sjöstrand, S. Mrenna and P.Z. Skands, A brief introduction to PYTHIA 8.1, Comput. Phys. Commun. 178 (2008) 852 [arXiv:0710.3820] [INSPIRE].

[95] Y. Gershtein and S. Knapen, Trigger strategy for displaced muon pairs following the CMS phase II upgrades, Phys. Rev. D 101 (2020) 032003 [arXiv:1907.00007] [InSPIRE].

[96] A. Hook, S. Kumar, Z. Liu and R. Sundrum, High quality QCD axion and the LHC, Phys. Rev. Lett. 124 (2020) 221801 [arXiv:1911.12364] [INSPIRE].

[97] A. Xiaocong, ACTS: a common tracking software, talk given at the PS Division of Particles E3 Fields Meeting (DPF2019), July 29-August 2, Boston, U.S.A. (2019).

[98] V.M.M. Cairo, Tracking and vertexing: challenges ahead, talk given at the LHC West Coast Jamboree, October 22-23, SLAC, Stanford, U.S.A. (2019).

[99] CMS collaboration, First level track jet trigger for displaced jets at high luminosity LHC, CMS-PAS-FTR-18-018 (2018).

[100] D. Contardo, M. Klute, J. Mans, L. Silvestris and J. Butler, Technical proposal for the Phase-II upgrade of the CMS detector, CERN-LHCC-2015-010 (2015).

[101] CMS collaboration, The CMS Level-1 tau lepton and Vector Boson Fusion triggers for the LHC Run II, CMS-CR-2017-346 (2017).

[102] ATLAS collaboration, Search for displaced vertices arising from decays of new heavy particles in $7 \mathrm{TeV}$ pp collisions at ATLAS, Phys. Lett. B 707 (2012) 478 [arXiv: 1109.2242] [INSPIRE].

[103] J. Liu, Z. Liu and L.-T. Wang, Enhancing long-lived particles searches at the LHC with precision timing information, Phys. Rev. Lett. 122 (2019) 131801 [arXiv:1805.05957] [INSPIRE]. 